\title{
A RIEMANNIAN FLETCHER-REEVES CONJUGATE GRADIENT METHOD FOR DOUBLY STOCHASTIC INVERSE EIGENVALUE PROBLEMS*
}

\author{
TENG-TENG $\mathrm{YAO}^{\dagger}$, ZHENG-JIAN BAI ${ }^{\ddagger}, \mathrm{ZHI} \mathrm{ZHAO}^{\S}$, AND WAI-KI CHING $₫$
}

\begin{abstract}
We consider the inverse eigenvalue problem of reconstructing a doubly stochastic matrix from the given spectrum data. We reformulate this inverse problem as a constrained nonlinear least squares problem over several matrix manifolds, which minimizes the distance between isospectral matrices and doubly stochastic matrices. Then a Riemannian Fletcher-Reeves conjugate gradient method is proposed for solving the constrained nonlinear least squares problem, and its global convergence is established. An extra gain is that a new Riemannian isospectral flow method is obtained. Our method is also extended to the case of prescribed entries. Finally, some numerical tests are reported to illustrate the efficiency of the proposed method.
\end{abstract}

Key words. inverse eigenvalue problem, doubly stochastic matrix, Riemannian Fletcher-Reeves conjugate gradient method, Riemannian isospectral flow

AMS subject classifications. 65F18, 65F15, 15A18, 65K05, 90C26, 90C48

DOI. $10.1137 / 15 \mathrm{M} 1023051$

1. Introduction. An $n$-by- $n$ real matrix $A$ is called a nonnegative matrix if all its entries are nonnegative, i.e., $A_{i j} \geq 0$ for all $i, j=1, \ldots, n$, where $A_{i j}$ means the $(i, j)$ th entry of $A$. An $n$-by- $n$ real matrix $A$ is called a (row) stochastic matrix if it is nonnegative with each row summing to 1 , i.e., $\sum_{j=1}^{n} A_{i j}=1$ for all $i=1, \ldots, n$. An $n$-by- $n$ real matrix $A$ is called a doubly stochastic matrix if it is nonnegative with each row and column summing to 1 , i.e., $\sum_{i=1}^{n} A_{i j}=\sum_{j=1}^{n} A_{i j}=1$ for all $i, j=1, \ldots, n$. Stochastic matrices and doubly stochastic matrices arise in various applications such as probability theory, statistics, quantum mechanics, hypergroups, economics, computer science, graph theory, physical chemistry, and population genetics, etc. See for instance $[8,11,15,16,18,22,24,29,33]$ and references therein.

This paper is concerned with the following doubly stochastic inverse eigenvalue problem (DSIEP).

DSIEP. Given a self-conjugate set of complex numbers $\left\{\lambda_{1}, \lambda_{2}, \ldots, \lambda_{n}\right\}$, find an $n$-by-n doubly stochastic matrix $C$ such that its eigenvalues are $\lambda_{1}, \lambda_{2}, \ldots, \lambda_{n}$ exactly.

In probability and combinatorics, the DSIEP aims to construct a special transition matrix (i.e., a doubly stochastic matrix) from the given spectral data. There exist

* Received by the editors May 26, 2015; accepted for publication (in revised form) by C.-H. Guo December 14, 2015; published electronically February 25, 2016.

http://www.siam.org/journals/simax/37-1/M102305.html

†School of Mathematical Sciences, Xiamen University, Xiamen 361005, People’s Republic of China (yaotengteng718@163.com).

${ }^{\ddagger}$ Corresponding author. School of Mathematical Sciences, Xiamen University, Xiamen 361005, People's Republic of China (zjbai@xmu.edu.cn). The research of this author was partially supported by the National Natural Science Foundation of China (11271308) and the Fundamental Research Funds for the Central Universities (20720150001).

$\S$ Department of Mathematics, Hangzhou Dianzi University, Hangzhou, People's Republic of China (zhaozhi231@163.com).

IAdvanced Modeling and Applied Computing Laboratory, Department of Mathematics, The University of Hong Kong, Hong Kong (wching@hku.hk). The research of this author was supported in part by the Research Grants Council of Hong Kong under grant 17301214, HKU CRCG grants, and the HKU Strategic Research Theme Fund on Computational Science.

215 
some solvability conditions for the DSIEP (see for instance [13, 14, 17, 19, 20, 23, 34] and references therein). However, there exist only a few numerical methods for solving the DSIEP. In particular, an idempotent system-based constructive method was proposed in [23] and Soules basis-based constructive methods were given in [21, 32]. An algorithm based on Householder transformations and rank 1 updating was presented in [39]. A fast Fourier transformation-based method was proposed in [27, 28]. However, all these constructive methods produce particular doubly stochastic matrices numerically under some special sufficient conditions.

Recently, there have been some Riemannian optimization methods for eigenproblems, which include a truncated conjugate gradient method for the symmetric generalized eigenvalue problem [1], a Riemannian trust-region method for the symmetric generalized eigenproblem [6], Newton's method and the conjugate gradient method for the symmetric eigenvalue problem [31], and a Riemannian Newton method for nonlinear eigenvalue problems [37].

In this paper, we propose a Riemannian nonlinear conjugate gradient method for solving the DSIEP. By using a real Schur matrix decomposition, the DSIEP is reformulated as a constrained nonlinear least squares problem over several matrix manifolds, where the cost function aims to minimize the distance between isospectral matrices and doubly stochastic matrices. The basic geometric properties of these matrix manifolds are studied and the Riemannian gradient of the cost function is derived. Then we propose a Riemannian Fletcher-Reeves conjugate gradient method for solving the constrained nonlinear least squares problem. This is sparked by a modified Fletcher-Reeves method proposed by Zhang, Zhou, and Li [36] and the recent development on Riemannian conjugate gradient methods [25, 26, 31]. The global convergence of the proposed method is established. An extra gain is that our model yields a new Riemannian isospectral flow method (similar to the isospectral flow method in [10] for solving the inverse stochastic spectrum problem). The proposed method is also extended to the DSIEP with prescribed entries. Finally, some numerical experiments are reported to show that the proposed geometric methods are effective for solving DSIEPs.

Throughout the paper, we use the following notation. The symbol $A^{T}$ denotes the transpose of a matrix $A$. $I_{n}$ is the identity matrix of order $n$. Let $\mathbb{R}^{n \times n}$ and $\mathcal{O}(n)$ be the set of all $n$-by- $n$ real matrices and the set of all $n$-by- $n$ orthogonal matrices, respectively. For any two matrices $A, B \in \mathbb{R}^{n \times n}, A \odot B$ and $[A, B]:=A B-B A$ mean the Hadamard product and the Lie bracket product of $A$ and $B$, respectively. For a matrix $A \in \mathbb{R}^{n \times n}, \operatorname{diag}(A)$ is a diagonal matrix with the same diagonal entries as $A$. Denote by $\operatorname{tr}(A)$ the sum of the diagonal entries of a square matrix $A$. Define the index set $\mathcal{N}:=\{(i, j): i, j=1, \ldots, n\}$. For a matrix $A \in \mathbb{R}^{n \times n}$, we define skew $(A):=\frac{1}{2}\left(A-A^{T}\right)$.

The rest of this paper is organized as follows. In section 2 we review some preliminary results on Riemannian manifolds. In section 3 we present a Riemannian Fletcher-Reeves conjugate gradient method for solving the DSIEP. In section 4 the global convergence of the proposed method is established. In section 5 we consider some extensions. Finally, some numerical tests are reported in section 6 and some concluding remarks are given in section 7 .

2. Preliminaries. In this section, we review some necessary concepts and basic results regarding Riemannian manifolds. One may refer to $[2,4]$ for more discussions.

Let $\mathcal{M}$ be a $d$-dimensional manifold. A curve $c$ on $\mathcal{M}$ is defined as a smooth mapping from $\mathbb{R}$ to $\mathcal{M}$. Let $X \in \mathcal{M}$. Denote by $\mathfrak{F}_{X}(\mathcal{M})$ the set of all smooth real- 
valued functions defined on a neighborhood of $X$. A tangent vector $\xi_{X}$ to $\mathcal{M}$ at $X$ is defined as a mapping from $\mathfrak{F}_{X}(\mathcal{M})$ to $\mathbb{R}$ such that

$$
\xi_{X} f=\dot{c}(0) f:=\left.\frac{d(f(c(t)))}{d t}\right|_{t=0} \quad \forall f \in \mathfrak{F}_{X}(\mathcal{M}),
$$

where $c: \mathbb{R} \rightarrow \mathcal{M}$ with $c(0)=X$ is any curve on $\mathcal{M}$ that realizes the tangent vector $\xi_{X}$. Let $T_{X} \mathcal{M}$ be the tangent space to $\mathcal{M}$ at $X$, which consists of all tangent vectors to $\mathcal{M}$ at $X$. Denote by $T \mathcal{M}$ the tangent bundle of $\mathcal{M}$ :

$$
T \mathcal{M}:=\bigcup_{X \in \mathcal{M}} T_{X} \mathcal{M}
$$

A vector field on $\mathcal{M}$ is a smooth function $\xi: \mathcal{M} \rightarrow T \mathcal{M}$, which assigns a tangent vector $\xi_{X} \in T_{X} \mathcal{M}$ to each point $X \in \mathcal{M}$. A Riemannian metric $g$ on $\mathcal{M}$ is a family of (positive definite) inner products

$$
g_{X}: T_{X} \mathcal{M} \times T_{X} \mathcal{M} \rightarrow \mathbb{R}, \quad X \in \mathcal{M},
$$

such that, for all smooth vector fields $\xi, \zeta$ on $\mathcal{M}, X \rightarrow g_{X}\left(\xi_{X}, \zeta_{X}\right)$ is a smooth function. The inner product $g_{X}(\cdot, \cdot)$ induces a norm $\left\|\xi_{X}\right\|=\sqrt{g_{X}\left(\xi_{X}, \xi_{X}\right)}$ on $T_{X} \mathcal{M}$. In this case, $(\mathcal{M}, g)$ is called a Riemannian manifold [4, p. 45]. In addition, if $\mathcal{M}$ is an embedded submanifold of a Riemannian manifold $(\overline{\mathcal{M}}, \bar{g})$, then $\mathcal{M}$ is also a Riemannian manifold if it is endowed with the Riemannian metric

$$
g_{X}\left(\xi_{X}, \zeta_{X}\right):=\bar{g}_{X}\left(\xi_{X}, \zeta_{X}\right), \quad \xi_{X}, \zeta_{X} \in T_{X} \mathcal{M},
$$

where $\xi_{X}$ and $\zeta_{X}$ are viewed as elements in $T_{X} \overline{\mathcal{M}}$.

Let $\mathcal{M}$ and $\mathcal{N}$ be two manifolds. Suppose that $F: \mathcal{M} \rightarrow \mathcal{N}$ is a smooth mapping. Then the differential (or derivative) $\mathrm{D} F(X)$ of $F$ at $X \in \mathcal{M}$ is defined as a mapping from $T_{X} \mathcal{M}$ to $T_{F(X)} \mathcal{N}$ such that

$$
\mathrm{D} F(X)\left[\xi_{X}\right] \in T_{F(X)} \mathcal{N} \quad \forall \xi_{X} \in T_{X} \mathcal{M},
$$

where the mapping $\mathrm{D} F(X)\left[\xi_{X}\right]$ from $\mathfrak{F}_{F(X)}(\mathcal{N})$ to $\mathbb{R}$ is defined by

$$
\mathrm{D} F(X)\left[\xi_{X}\right] f=\xi_{X}(f \circ F) \quad \forall f \in \mathfrak{F}_{F(X)}(\mathcal{N}) .
$$

Let $\bar{F}: \overline{\mathcal{M}} \rightarrow \mathcal{N}$ be a smooth mapping between two Riemannian manifolds $\overline{\mathcal{M}}$ and $\mathcal{N}$. Suppose that $F$ is the restriction of $\bar{F}$ to an embedded Riemannian submanifold $\mathcal{M}$. According to (2.1) and (2.2), it follows that

$$
\begin{aligned}
\mathrm{D} F(X)\left[\xi_{X}\right] f & =\xi_{X}(f \circ F)=\left.\frac{d(f(F(c(t))))}{d t}\right|_{t=0}=\left.\frac{d(f(\bar{F}(c(t))))}{d t}\right|_{t=0} \\
& =\xi_{X}(f \circ \bar{F})=\mathrm{D} \bar{F}(X)\left[\xi_{X}\right] f \quad \forall f \in \mathfrak{F}_{F(X)}(\mathcal{N})
\end{aligned}
$$

where $c: \mathbb{R} \rightarrow \mathcal{M}$ is a curve in $\mathcal{M}$ with $c(0)=X$ and $\dot{c}(0)=\xi_{X}$. Thus, we have

$$
\mathrm{D} F(X)\left[\xi_{X}\right]=\mathrm{D} \bar{F}(X)\left[\xi_{X}\right] \quad \forall \xi_{X} \in T_{X} \mathcal{M}, X \in \mathcal{M} .
$$

Suppose that $(\mathcal{M}, g)$ is a Riemannian manifold and $f: \mathcal{M} \rightarrow \mathbb{R}$ is a smooth function. Then the Riemannian gradient $\operatorname{grad} f(X)$ of $f$ at $X \in \mathcal{M}$ is defined as the unique element in $T_{X} \mathcal{M}$ such that

$$
g_{X}\left(\operatorname{grad} f(X), \xi_{X}\right)=\mathrm{D} f(X)\left[\xi_{X}\right] \quad \forall \xi_{X} \in T_{X} \mathcal{M} .
$$

Copyright (c) by SIAM. Unauthorized reproduction of this article is prohibited. 
Finally, we recall the definition of pullback. We need the concept of retraction, which was originally introduced in the field of algebraic topology [12]. Here, we use the definition of retraction in [4, 5, 30]. For a smooth mapping $F: \mathcal{M} \rightarrow \mathcal{N}$ between two manifolds $\mathcal{M}$ and $\mathcal{N}$ and a retraction $R$ on $\mathcal{M}$, the pullback $\widehat{F}$ of $F$ is a mapping from $T \mathcal{M}$ to $\mathcal{N}$ defined by

$$
\widehat{F}(\xi):=F(R(\xi)) \quad \forall \xi \in T \mathcal{M} .
$$

Let $\widehat{F}_{X}$ denote the restriction of $\widehat{F}$ to $T_{X} \mathcal{M}$, which is defined by

$$
\widehat{F}_{X}\left(\xi_{X}\right)=F\left(R_{X}\left(\xi_{X}\right)\right) \quad \forall \xi_{X} \in T_{X} \mathcal{M} .
$$

Then we have, by the definition of retraction [4],

$$
\mathrm{D} F(X)\left[\xi_{X}\right]=\mathrm{D} F\left(R_{X}\left(0_{X}\right)\right)\left[\xi_{X}\right]=\mathrm{D} F\left(R_{X}\left(0_{X}\right)\right)\left[\mathrm{D} R_{X}\left(0_{X}\right)\left[\xi_{X}\right]\right]=\mathrm{D} \widehat{F}_{X}\left(0_{X}\right)\left[\xi_{X}\right]
$$

for any $X \in \mathcal{M}$ and $\xi_{X} \in T_{X} \mathcal{M}$. Thus,

$$
\mathrm{D} F(X)=\mathrm{D} \widehat{F}_{X}\left(0_{X}\right), \quad X \in \mathcal{M} .
$$

3. Doubly stochastic inverse eigenvalue problem. In this section, we propose a Riemannian Fletcher-Reeves conjugate gradient method for solving the DSIEP. The DSIEP is turned into a nonlinear least squares problem defined on some matrix manifolds. Then some basic geometric properties of these matrix manifolds are studied. Finally, a Riemannian Fletcher-Reeves conjugate gradient method is proposed for solving the nonlinear least squares problem.

3.1. Reformulation. Since the set of complex numbers $\left\{\lambda_{1}, \lambda_{2}, \ldots, \lambda_{n}\right\}$ is closed under complex conjugation, without loss of generality, one may assume

$\lambda_{2 j-1}=a_{j}+b_{j} \sqrt{-1}, \quad \lambda_{2 j}=a_{j}-b_{j} \sqrt{-1}, \quad j=1, \ldots, s ; \quad \lambda_{j} \in \mathbb{R}, \quad j=2 s+1, \ldots, n$, where $a_{j}, b_{j} \in \mathbb{R}$ and $b_{j} \neq 0$ for $j=1, \ldots, s$. Define a block diagonal matrix $\Lambda$ by

$$
\Lambda:=\operatorname{blkdiag}\left(\lambda_{1}^{[2]}, \ldots, \lambda_{s}^{[2]}, \lambda_{2 s+1}, \ldots, \lambda_{n}\right)
$$

with diagonal blocks $\lambda_{1}^{[2]}, \ldots, \lambda_{s}^{[2]}, \lambda_{2 s+1}, \ldots, \lambda_{n}$, where

$$
\lambda_{j}^{[2]}=\left[\begin{array}{cc}
a_{j} & b_{j} \\
-b_{j} & a_{j}
\end{array}\right], \quad j=1, \ldots, s .
$$

Define the set $\mathcal{U}$ by

$$
\mathcal{U}:=\left\{U \in \mathbb{R}^{n \times n} \mid U_{i j}=0,(i, j) \in \mathcal{I}\right\},
$$

where $\mathcal{I}$ is the index subset:

$$
\mathcal{I}:=\left\{(i, j) \mid i \geq j \text { or } \Lambda_{i j} \neq 0, i, j=1, \ldots, n\right\} .
$$

Define by $\mathcal{Z}$ the set of all $n$-by- $n$ doubly stochastic matrices, i.e.,

$$
\mathcal{Z}:=\left\{Z \odot Z \in \mathbb{R}^{n \times n} \mid Z \in \mathcal{O B}, Z^{T} \in \mathcal{O B}\right\},
$$

Copyright (c) by SIAM. Unauthorized reproduction of this article is prohibited. 
where the set $\mathcal{O B}$ is the oblique manifold $[3,4]$ :

$$
\mathcal{O B}:=\left\{Z \in \mathbb{R}^{n \times n} \mid \operatorname{diag}\left(Z Z^{T}\right)=I_{n}\right\}
$$

Then one may define a smooth manifold of isospectral matrices by

$$
\mathcal{W}(\Lambda):=\left\{X \in \mathbb{R}^{n \times n} \mid X=Q(\Lambda+U) Q^{T}, Q \in \mathcal{O}(n), U \in \mathcal{U}\right\} .
$$

The DSIEP has a solution if and only if $\mathcal{W}(\Lambda) \cap \mathcal{Z} \neq \emptyset$.

We assume that the DSIEP has at least one solution. Then the DSIEP aims to find a solution to the following nonlinear matrix equation:

$$
H(Z, Q, U):=\left(H_{1}(Z, Q, U), H_{2}(Z)\right)=0
$$

for $(Z, Q, U) \in \mathcal{O B} \times \mathcal{O}(n) \times \mathcal{U}$. The mappings $H_{1}: \mathcal{O B} \times \mathcal{O}(n) \times \mathcal{U} \rightarrow \mathbb{R}^{n \times n}$ and $H_{2}: \mathcal{O B} \rightarrow \mathbb{R}^{n}$ are defined by

$$
\begin{cases}H_{1}(Z, Q, U)=Z \odot Z-Q(\Lambda+U) Q^{T}, & (Z, Q, U) \in \mathcal{O B} \times \mathcal{O}(n) \times \mathcal{U}, \\ H_{2}(Z)=(Z \odot Z)^{T} \mathbf{e}-\mathbf{e}, & Z \in \mathcal{O B},\end{cases}
$$

where $\mathbf{e} \in \mathbb{R}^{n}$ is an $n$-vector of all ones.

It is easy to see that if $(\bar{Z}, \bar{Q}, \bar{U}) \in \mathcal{O B} \times \mathcal{O}(n) \times \mathcal{U}$ is a solution to $H(Z, Q, U)=0$, then $\bar{C}=\bar{Z} \odot \bar{Z}$ is a solution to the DSIEP. Alternatively, one may solve the following nonlinear least squares problem:

$$
\begin{array}{ll}
\min & h(Z, Q, U):=\frac{1}{2}\|H(Z, Q, U)\|_{F}^{2} \\
\text { subject to (s.t.) } & Z \in \mathcal{O B}, \quad Q \in \mathcal{O}(n), \quad U \in \mathcal{U},
\end{array}
$$

where $\|\cdot\|_{F}$ is the matrix Frobenius norm.

3.2. Basic properties. In this section, we give some basic geometric properties of the product manifold $\mathcal{O B} \times \mathcal{O}(n) \times \mathcal{U}$. We first note that the dimensions of $\mathcal{O B}$, $\mathcal{O}(n)$, and $\mathcal{U}$ are given by $[4$, p. 27$]$

$$
\operatorname{dim}(\mathcal{O B})=n(n-1), \quad \operatorname{dim}(\mathcal{O}(n))=\frac{1}{2} n(n-1), \quad \operatorname{dim}(\mathcal{U})=|\mathcal{J}|,
$$

where $|\mathcal{J}|$ is the cardinality of the index subset $\mathcal{J}:=\mathcal{N} \backslash \mathcal{I}$. Then we have

$$
\operatorname{dim}(\mathcal{O B} \times \mathcal{O}(n) \times \mathcal{U})=n(n-1)+\frac{n(n-1)}{2}+|\mathcal{J}|
$$

It follows that

$$
\operatorname{dim}(\mathcal{O B} \times \mathcal{O}(n) \times \mathcal{U})>\operatorname{dim}\left(\mathbb{R}^{n \times n}\right)+\operatorname{dim}\left(\mathbb{R}^{n}\right) \quad \text { for } n \geq 4
$$

Hence, the nonlinear equation $H(Z, Q, U)=0$ is an underdetermined system defined from the product manifold $\mathcal{O B} \times \mathcal{O}(n) \times \mathcal{U}$ to the Euclidean space $\mathbb{R}^{n \times n}$ for $n \geq 4$.

The tangent space of $\mathcal{O B} \times \mathcal{O}(n) \times \mathcal{U}$ at $(Z, Q, U) \in \mathcal{O B} \times \mathcal{O}(n) \times \mathcal{U}$ is given by

$$
T_{(Z, Q, U)} \mathcal{O B} \times \mathcal{O}(n) \times \mathcal{U}=T_{Z} \mathcal{O B} \times T_{Q} \mathcal{O}(n) \times T_{U} \mathcal{U},
$$


where $T_{Z} \mathcal{O B}, T_{Q} \mathcal{O}(n)$, and $T_{U} \mathcal{U}$ are the tangent spaces of $\mathcal{O B}, \mathcal{O}(n)$, and $\mathcal{U}$ at $Z \in \mathcal{O B}, Q \in \mathcal{O}(n)$, and $U \in \mathcal{U}$, respectively ([3] and [4, p. 42]):

$$
\left\{\begin{aligned}
T_{Z} \mathcal{O B} & =\left\{W \in \mathbb{R}^{n \times n} \mid \operatorname{diag}\left(Z W^{T}\right)=0\right\}, \\
T_{Q} \mathcal{O}(n) & =\left\{Q K \mid K^{T}=-K, K \in \mathbb{R}^{n \times n}\right\}, \\
T_{U} \mathcal{U} & =\mathcal{U} .
\end{aligned}\right.
$$

We now define a Riemannian metric on $\mathcal{O B} \times \mathcal{O}(n) \times \mathcal{U}$. Let the Euclidean space $\mathbb{R}^{n \times n} \times \mathbb{R}^{n \times n} \times \mathbb{R}^{n \times n}$ be equipped with the following natural inner product

$$
\left\langle\left(Z_{1}, Q_{1}, U_{1}\right),\left(Z_{2}, Q_{2}, U_{2}\right)\right\rangle:=\operatorname{tr}\left(Z_{1}^{T} Z_{2}\right)+\operatorname{tr}\left(Q_{1}^{T} Q_{2}\right)+\operatorname{tr}\left(U_{1}^{T} U_{2}\right)
$$

for all $\left(Z_{1}, Q_{1}, U_{1}\right),\left(Z_{2}, Q_{2}, U_{2}\right) \in \mathbb{R}^{n \times n} \times \mathbb{R}^{n \times n} \times \mathbb{R}^{n \times n}$ and its induced norm $\|\cdot\|$. It is obvious that $\mathcal{O B} \times \mathcal{O}(n) \times \mathcal{U}$ is an embedded submanifold of the Euclidean space $\mathbb{R}^{n \times n} \times \mathbb{R}^{n \times n} \times \mathbb{R}^{n \times n}$. Thus, $\mathcal{O B} \times \mathcal{O}(n) \times \mathcal{U}$ can be equipped with a induced Riemannian metric:

$$
g_{(Z, Q, U)}\left(\left(\xi_{1}, \zeta_{1}, \eta_{1}\right),\left(\xi_{2}, \zeta_{2}, \eta_{2}\right)\right):=\left\langle\left(\xi_{1}, \zeta_{1}, \eta_{1}\right),\left(\xi_{2}, \zeta_{2}, \eta_{2}\right)\right\rangle
$$

for all $(Z, Q, U) \in \mathcal{O B} \times \mathcal{O}(n) \times \mathcal{U}$ and $\left(\xi_{1}, \zeta_{1}, \eta_{1}\right),\left(\xi_{2}, \zeta_{2}, \eta_{2}\right) \in T_{(Z, Q, U)} \mathcal{O B} \times \mathcal{O}(n) \times \mathcal{U}$. Without causing any confusion, in what follows, let $\langle\cdot, \cdot\rangle$ and $\|\cdot\|$ stand for the Riemannian metric on $\mathcal{O B} \times \mathcal{O}(n) \times \mathcal{U}$ and its induced norm. Therefore, $(\mathcal{O B} \times$ $\mathcal{O}(n) \times \mathcal{U}, g)$ is a Riemannian manifold. Then the orthogonal projections of any given points $\xi, \zeta, \eta \in \mathbb{R}^{n \times n}$ onto $T_{Z} \mathcal{O B}, T_{Q} \mathcal{O}(n)$, and $T_{U} \mathcal{U}$ are given, respectively, by ([3] and $[4$, p. 48])

$$
\Pi_{Z} \xi=\xi-\operatorname{diag}\left(Z \xi^{T}\right) Z, \quad \Pi_{Q} \zeta=Q \operatorname{skew}\left(Q^{T} \zeta\right), \quad \Pi_{U} \eta=G \odot \eta,
$$

where the matrix $G \in \mathbb{R}^{n \times n}$ is defined by

$$
G_{i j}:= \begin{cases}0, & \text { if }(i, j) \in \mathcal{I} \\ 1, & \text { otherwise }\end{cases}
$$

Hence, the orthogonal projection of a point $(\xi, \zeta, \eta) \in \mathbb{R}^{n \times n} \times \mathbb{R}^{n \times n} \times \mathbb{R}^{n \times n}$ onto $T_{(Z, Q, U)} \mathcal{O B} \times \mathcal{O}(n) \times \mathcal{U}$ takes the form of

$$
\Pi_{(Z, Q, U)}(\xi, \zeta, \eta)=\left(\Pi_{Z} \xi, \Pi_{Q} \zeta, \Pi_{U} \eta\right) .
$$

Next, we give a retraction $R$ on $\mathcal{O B} \times \mathcal{O}(n) \times \mathcal{U}$. As in [3] and [4, p. 58], we can choose the retractions on $\mathcal{O B}, \mathcal{O}(n)$, and $\mathcal{U}$ as follows:

$$
\left\{\begin{array}{l}
R_{Z}\left(\xi_{Z}\right)=\left(\operatorname{diag}\left(\left(Z+\xi_{Z}\right)\left(Z+\xi_{Z}\right)^{T}\right)\right)^{-1 / 2}\left(Z+\xi_{Z}\right) \text { for } \xi_{Z} \in T_{Z} \mathcal{O B}, \\
R_{Q}\left(\zeta_{Q}\right)=\mathrm{qf}\left(Q+\zeta_{Q}\right) \text { for } \zeta_{Q} \in T_{Q} \mathcal{O}(n), \\
R_{U}\left(\eta_{U}\right)=U+\eta_{U} \text { for } \eta_{U} \in T_{U} \mathcal{U} .
\end{array}\right.
$$

Here, for a given nonsingular matrix $A \in \mathbb{R}^{n \times n}$, $\operatorname{qf}(A)$ means the $Q$ factor of the QR decomposition of $A$ in the form of $A=\widetilde{Q} \widetilde{R}$, where $\widetilde{Q} \in \mathcal{O}(n)$ and $\widetilde{R}$ is an upper triangular matrix with strictly positive diagonal elements. Therefore, a retraction $R$ on $\mathcal{O B} \times \mathcal{O}(n) \times \mathcal{U}$ can be defined by

$$
R_{(Z, Q, U)}\left(\xi_{Z}, \xi_{Q}, \xi_{U}\right)=\left(R_{Z}\left(\xi_{Z}\right), R_{Q}\left(\xi_{Q}\right), R_{U}\left(\xi_{U}\right)\right)
$$

Copyright $@$ ㅇ by SIAM. Unauthorized reproduction of this article is prohibited. 
for all $(Z, Q, U) \in \mathcal{O B} \times \mathcal{O}(n) \times \mathcal{U}$ and $\left(\xi_{Z}, \xi_{Q}, \xi_{U}\right) \in T_{(Z, Q, U)} \mathcal{O B} \times \mathcal{O}(n) \times \mathcal{U}$.

We now define a vector transport on $\mathcal{O B} \times \mathcal{O}(n) \times \mathcal{U}$. We use the concept of vector transport in [4, p. 169], which is related to the concept of parallel translation (see for instance [4, p. 104]) and is easy to implement numerically. We see that $\mathcal{O B}$, $\mathcal{O}(n)$, and $\mathcal{U}$ are embedded Riemannian submanifolds of $\mathbb{R}^{n \times n}$. By using orthogonal projections in (3.4), the vector transports on $\mathcal{O B}, \mathcal{O}(n)$, and $\mathcal{U}$ take the forms $[4$, p. 174]

$$
\left\{\begin{array}{l}
\mathcal{T}_{\eta_{Z}} \xi_{Z}=\Pi_{R_{Z}\left(\eta_{Z}\right)} \xi_{Z}=\xi_{Z}-\operatorname{diag}\left(R_{Z}\left(\eta_{Z}\right) \xi_{Z}^{T}\right) R_{Z}\left(\eta_{Z}\right) \quad \text { for } \xi_{Z}, \eta_{Z} \in T_{Z} \mathcal{O B}, \\
\mathcal{T}_{\eta_{Q}} \xi_{Q}=\Pi_{R_{Q}\left(\eta_{Q}\right)} \xi_{Q}=R_{Q}\left(\eta_{Q}\right) \operatorname{skew}\left(\left(R_{Q}\left(\eta_{Q}\right)\right)^{T} \xi_{Q}\right) \quad \text { for } \xi_{Q}, \eta_{Q} \in T_{Q} \mathcal{O}(n), \\
\mathcal{T}_{\eta_{U}} \xi_{U}=\Pi_{R_{U}\left(\eta_{U}\right)} \xi_{U}=\xi_{U} \quad \text { for } \xi_{U}, \eta_{U} \in T_{U} \mathcal{U} .
\end{array}\right.
$$

Therefore, by (3.5), the vector transport on $\mathcal{O B} \times \mathcal{O}(n) \times \mathcal{U}$ can be defined by

$$
\mathcal{T}_{\left(\eta_{Z}, \eta_{Q}, \eta_{U}\right)}\left(\xi_{Z}, \xi_{Q}, \xi_{U}\right):=\Pi_{R_{(Z, Q, U)}\left(\eta_{Z}, \eta_{Q}, \eta_{U}\right)}\left(\xi_{Z}, \xi_{Q}, \xi_{U}\right)=\left(\mathcal{T}_{\eta_{Z}} \xi_{Z}, \mathcal{T}_{\eta_{Q}} \xi_{Q}, \mathcal{T}_{\eta_{U}} \xi_{U}\right)
$$

for any $\left(\xi_{Z}, \xi_{Q}, \xi_{U}\right),\left(\eta_{Z}, \eta_{Q}, \eta_{U}\right) \in T_{(Z, Q, U)} \mathcal{O B} \times \mathcal{O}(n) \times \mathcal{U}$.

We now derive explicit expressions of the differential of $H$ defined in (3.1) and the Riemannian gradient of the cost function $h$ defined in (3.2). To do so, we define the mapping $\bar{H}: \mathbb{R}^{n \times n} \times \mathbb{R}^{n \times n} \times \mathbb{R}^{n \times n} \rightarrow \mathbb{R}^{n \times n} \times \mathbb{R}^{n}$ by

$$
\bar{H}(Z, Q, U)=\left(\bar{H}_{1}(Z, Q, U), \bar{H}_{2}(Z)\right):=\left(Z \odot Z-Q(\Lambda+U) Q^{T},(Z \odot Z)^{T} \mathbf{e}-\mathbf{e}\right)
$$

for all $(Z, Q, U) \in \mathbb{R}^{n \times n} \times \mathbb{R}^{n \times n} \times \mathbb{R}^{n \times n}$. Thus, $H$ is the restriction of $\bar{H}$ onto

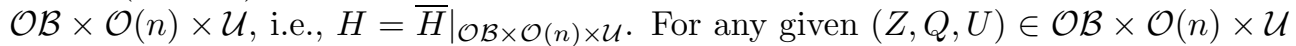
and $(\Delta Z, \Delta Q, \Delta U) \in T_{(Z, Q, U)} \mathcal{O B} \times \mathcal{O}(n) \times \mathcal{U}$, we get by (3.6),

$$
\begin{aligned}
& \bar{H}_{1}(Z+\Delta Z, Q+\Delta Q, U+\Delta U)-\bar{H}_{1}(Z, Q, U) \\
= & (Z+\Delta Z) \odot(Z+\Delta Z)-(Q+\Delta Q)(\Lambda+U+\Delta U)(Q+\Delta Q)^{T} \\
& \quad-\left(Z \odot Z-Q(\Lambda+U) Q^{T}\right) \\
= & 2 Z \odot \Delta Z-Q(\Lambda+U)(\Delta Q)^{T}-\Delta Q(\Lambda+U) Q^{T} \\
& \quad-\Delta Q(\Lambda+U)(\Delta Q)^{T}-Q \Delta U Q^{T}+\Delta Z \odot \Delta Z-Q \Delta U(\Delta Q)^{T} \\
& \quad-\Delta Q \Delta U Q^{T}-\Delta Q \Delta U(\Delta Q)^{T} \\
= & 2 Z \odot \Delta Z-Q(\Lambda+U) Q^{T}\left(\Delta Q Q^{T}\right)^{T}-\left(\Delta Q Q^{T}\right) Q(\Lambda+U) Q^{T}-Q \Delta U Q^{T} \\
& +\Delta Z \odot \Delta Z-\Delta Q(\Lambda+U)(\Delta Q)^{T}-Q \Delta U(\Delta Q)^{T}-\Delta Q \Delta U Q^{T}-\Delta Q \Delta U(\Delta Q)^{T} \\
= & 2 Z \odot \Delta Z+\left[Q(\Lambda+U) Q^{T}, \Delta Q Q^{T}\right]-Q \Delta U Q^{T} \\
& +\Delta Z \odot \Delta Z-\Delta Q(\Lambda+U)(\Delta Q)^{T}-Q \Delta U(\Delta Q)^{T}-\Delta Q \Delta U Q^{T}-\Delta Q \Delta U(\Delta Q)^{T}
\end{aligned}
$$

and

$$
\begin{aligned}
& \bar{H}_{2}(Z+\Delta Z)-\bar{H}_{2}(Z) \\
= & ((Z+\Delta Z) \odot(Z+\Delta Z))^{T} \mathbf{e}-(Z \odot Z)^{T} \mathbf{e} \\
= & 2(Z \odot \Delta Z)^{T} \mathbf{e}+(\Delta Z \odot \Delta Z)^{T} \mathbf{e},
\end{aligned}
$$

where the condition $\left(\Delta Q Q^{T}\right)^{T}=-\Delta Q Q^{T}$ is used. Hence, by using (2.3), the differential $\mathrm{D} H(Z, Q, U): T_{(Z, Q, U)} \mathcal{O B} \times \mathcal{O}(n) \times \mathcal{U} \rightarrow T_{H(Z, Q, U)} \mathbb{R}^{n \times n} \times \mathbb{R}^{n} \simeq \mathbb{R}^{n \times n} \times \mathbb{R}^{n}$ 
of $H$ at a point $(Z, Q, U) \in \mathcal{O B} \times \mathcal{O}(n) \times \mathcal{U}$ is determined by

$$
\begin{aligned}
& \mathrm{D} H(Z, Q, U)[(\Delta Z, \Delta Q, \Delta U)]=\left(\mathrm{DH}_{1}(Z, Q, U)[(\Delta Z, \Delta Q, \Delta U)], \mathrm{D} H_{2}(Z)[(\Delta Z])\right. \\
= & \left(2 Z \odot \Delta Z+\left[Q(\Lambda+U) Q^{T}, \Delta Q Q^{T}\right]-Q \Delta U Q^{T}, 2(Z \odot \Delta Z)^{T} \mathbf{e}\right)
\end{aligned}
$$

for all $(\Delta Z, \Delta Q, \Delta U) \in T_{(Z, Q, U)} \mathcal{O B} \times \mathcal{O}(n) \times \mathcal{U}$. The adjoint $(\mathrm{D} H(Z, Q, U))^{*}$ : $T_{H(Z, Q, U)} \mathbb{R}^{n \times n} \times \mathbb{R}^{n} \rightarrow T_{(Z, Q, U)} \mathcal{O B} \times \mathcal{O}(n) \times \mathcal{U}$ of the operator $\mathrm{D} H(Z, Q, U)$ is determined by

$$
\begin{aligned}
& \left\langle\left(\Delta W_{1}, \Delta W_{2}\right), \mathrm{D} H(Z, Q, U)[(\Delta Z, \Delta Q, \Delta U)]\right\rangle_{F} \\
= & g\left((\mathrm{D} H(Z, Q, U))^{*}\left[\left(\Delta W_{1}, \Delta W_{2}\right)\right],(\Delta Z, \Delta Q, \Delta U)\right)
\end{aligned}
$$

for all $(\Delta Z, \Delta Q, \Delta U) \in T_{(Z, Q, U)} \mathcal{O B} \times \mathcal{O}(n) \times \mathcal{U}$ and $\left(\Delta W_{1}, \Delta W_{2}\right) \in T_{H(Z, Q, U)} \mathbb{R}^{n \times n} \times$ $\mathbb{R}^{n}$, where $\langle\cdot, \cdot\rangle_{F}$ is the Frobenius inner product on $\mathbb{R}^{n \times n} \times \mathbb{R}^{n}$. Thus,

$$
\begin{aligned}
& (\mathrm{D} H(Z, Q, U))^{*}[\Delta W] \\
= & \left((\mathrm{D} H(Z, Q, U))_{1}^{*}[\Delta W],(\mathrm{D} H(Z, Q, U))_{2}^{*}[\Delta W],(\mathrm{D} H(Z, Q, U))_{3}^{*}[\Delta W]\right)
\end{aligned}
$$

for all $\Delta W:=\left(\Delta W_{1}, \Delta W_{2}\right) \in T_{H(Z, Q, U)} \mathbb{R}^{n \times n} \times \mathbb{R}^{n}$, where for each $\Delta W:=\left(\Delta W_{1}\right.$, $\left.\Delta W_{2}\right) \in T_{H(Z, Q, U)} \mathbb{R}^{n \times n} \times \mathbb{R}^{n}$,

$$
\left\{\begin{aligned}
(\mathrm{D} H(Z, Q, U))_{1}^{*}[\Delta W]= & 2 Z \odot\left(\Delta W_{1}+\mathbf{e}\left(\Delta W_{2}\right)^{T}\right) \\
& -2 \operatorname{diag}\left(Z\left(Z \odot\left(\Delta W_{1}+\mathbf{e}\left(\Delta W_{2}\right)^{T}\right)\right)^{T}\right) Z, \\
(\mathrm{D} H(Z, Q, U))_{2}^{*}[\Delta W]= & \frac{1}{2}\left(\left[Q(\Lambda+U) Q^{T},\left(\Delta W_{1}\right)^{T}\right]+\left[Q(\Lambda+U)^{T} Q^{T}, \Delta W_{1}\right]\right) Q, \\
(\mathrm{D} H(Z, Q, U))_{3}^{*}[\Delta W]= & -G \odot\left(Q^{T} \Delta W_{1} Q\right) .
\end{aligned}\right.
$$

By using (3.7), the Riemannian gradient of $h$ at a point $(Z, Q, U) \in \mathcal{O B} \times \mathcal{O}(n) \times \mathcal{U}$ is given by $[4$, p. 184]

$$
\begin{aligned}
& \operatorname{grad} h(Z, Q, U)=(\mathrm{D} H(Z, Q, U))^{*}[H(Z, Q, U)] \\
&:=\quad\left((\mathrm{D} H)_{1}^{*}[H(Z, Q, U)],(\mathrm{D} H)_{2}^{*}[H(Z, Q, U)],(\mathrm{D} H)_{3}^{*}[H(Z, Q, U)]\right),
\end{aligned}
$$

where

$$
\left\{\begin{aligned}
(\mathrm{D} H)_{1}^{*}[H(Z, Q, U)]= & 2 Z \odot\left(H_{1}(Z, Q, U)+\mathbf{e} H_{2}^{T}(Z, Q, U)\right) \\
& -2 \operatorname{diag}\left(Z\left(Z \odot\left(H_{1}(Z, Q, U)+\mathbf{e} H_{2}^{T}(Z, Q, U)\right)\right)^{T}\right) Z, \\
(\mathrm{D} H)_{2}^{*}[H(Z, Q, U)]= & \frac{1}{2}\left[Q(\Lambda+U) Q^{T}, H_{1}^{T}(Z, Q, U)\right] Q \\
& +\frac{1}{2}\left[Q(\Lambda+U)^{T} Q^{T}, H_{1}(Z, Q, U)\right] Q \\
(\mathrm{D} H)_{3}^{*}[H(Z, Q, U)]= & -G \odot\left(Q^{T} H_{1}(Z, Q, U) Q\right) .
\end{aligned}\right.
$$

By using a similar idea in [9] and [10], a Riemannian gradient flow method for the cost function $h$ over $\mathcal{O B} \times \mathcal{O}(n) \times \mathcal{U}$ is given by

$$
\frac{d(Z, Q, U)}{d t}=-\operatorname{grad} h(Z, Q, U)
$$

Given a starting point $(Z(0), Q(0), U(0)) \in \mathcal{O B} \times \mathcal{O}(n) \times \mathcal{U}$, this yields an initial value problem for the DSIEP (3.2). Then one may find a solution to the DSIEP

Copyright $@$ by SIAM. Unauthorized reproduction of this article is prohibited. 
(3.2) by using existing ODE solvers for the differential equation (3.9). The numerical experiments in section 6 show that this extra isospectral flow method is effective for small- and medium-scale problems.

Finally, as in (2.4), the pullback mappings $\widehat{H}: T \mathcal{O B} \times \mathcal{O}(n) \times \mathcal{U} \rightarrow \mathbb{R}^{n \times n} \times \mathbb{R}^{n}$ and $\widehat{h}: T \mathcal{O B} \times \mathcal{O}(n) \times \mathcal{U} \rightarrow \mathbb{R}$ of $H$ and $h$ through the retraction $R$ on $\mathcal{O B} \times \mathcal{O}(n) \times \mathcal{U}$ are given, respectively, by

$$
\widehat{H}(\xi, \zeta, \eta)=H(R(\xi, \zeta, \eta)) \text { and } \widehat{h}(\xi, \zeta, \eta)=h(R(\xi, \zeta, \eta))
$$

for all $(\xi, \zeta, \eta) \in T \mathcal{O B} \times \mathcal{O}(n) \times \mathcal{U}$, where $\widehat{H}_{(Z, Q, U)}=\left.\widehat{H}\right|_{T_{(Z, Q, U)} \mathcal{O} B \times \mathcal{O}(n) \times \mathcal{U}}$ and

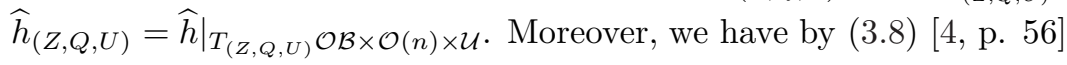

$\operatorname{grad} h(Z, Q, U)=\operatorname{grad} \widehat{h}_{(Z, Q, U)}\left(0_{(Z, Q, U)}\right)=\left(\mathrm{D} \widehat{H}_{(Z, Q, U)}\left(0_{(Z, Q, U)}\right)\right)^{*}\left[\widehat{H}_{(Z, Q, U)}\left(0_{(Z, Q, U)}\right)\right]$, where $0_{(Z, Q, U)}$ is the origin of $T_{(Z, Q, U)} \mathcal{O B} \times \mathcal{O}(n) \times \mathcal{U}$.

3.3. A Riemannian Fletcher-Reeves conjugate gradient method. In this section, we present a Riemannian Fletcher-Reeves conjugate gradient method for solving the DSIEP (3.2). This is motivated by a modified Fletcher-Reeves method with Armijo-type line search in [36], which always provides a descent direction of the objective function, while the direction generated by a standard Fletcher-Reeves method with Armijo-type line search or Wolfe-type line search is not necessarily one of descent (see [36]). The corresponding geometric algorithm is described as follows.

Algorithm 3.1 (A Riemannian Fletcher-Reeves conjugate gradient method).

Step 0. Given $\left(Z^{0}, Q^{0}, U^{0}\right) \in \mathcal{O B} \times \mathcal{O}(n) \times \mathcal{U}, \alpha \geq 1, \rho, \delta_{1} \in(0,1), \delta_{2}>0 . k:=0$.

Step 1. If $h\left(Z^{k}, Q^{k}, U^{k}\right)=0$, then stop. Otherwise, go to Step 2.

Step 2. Set

$$
\begin{aligned}
& \left(\Delta Z_{k}, \Delta Q_{k}, \Delta U_{k}\right) \\
:= & -\operatorname{grad} h\left(Z^{k}, Q^{k}, U^{k}\right)+\beta_{k} \Delta Y_{k}-\theta_{k} \operatorname{grad} h\left(Z^{k}, Q^{k}, U^{k}\right),
\end{aligned}
$$

where

$$
\begin{aligned}
\Delta Y_{k}:=\mathcal{T}_{\alpha_{k-1}\left(\Delta Z_{k-1}, \Delta Q_{k-1}, \Delta U_{k-1}\right)}\left(\Delta Z_{k-1}, \Delta Q_{k-1}, \Delta U_{k-1}\right), \\
\beta_{k}:=\frac{\left\|\operatorname{grad} h\left(Z^{k}, Q^{k}, U^{k}\right)\right\|^{2}}{\left\|\operatorname{grad} h\left(Z^{k-1}, Q^{k-1}, U^{k-1}\right)\right\|^{2}}, \\
\theta_{k}:=\frac{\left\langle\operatorname{grad} h\left(Z^{k}, Q^{k}, U^{k}\right), \Delta Y_{k}\right\rangle}{\left\|\operatorname{grad} h\left(Z^{k-1}, Q^{k-1}, U^{k-1}\right)\right\|^{2}}
\end{aligned}
$$

Step 3. Determine $\alpha_{k}=\max \left\{\alpha \rho^{j}, j=0,1,2, \ldots\right\}$ such that

$$
\begin{aligned}
& h\left(R_{\left(Z^{k}, Q^{k}, U^{k}\right)}\left(\alpha_{k}\left(\Delta Z_{k}, \Delta Q_{k}, \Delta U_{k}\right)\right)\right)-h\left(Z^{k}, Q^{k}, U^{k}\right) \\
\leq & \delta_{1} \alpha_{k}\left\langle\operatorname{grad} h\left(Z^{k}, Q^{k}, U^{k}\right),\left(\Delta Z_{k}, \Delta Q_{k}, \Delta U_{k}\right)\right\rangle \\
& -\delta_{2} \alpha_{k}^{2}\left\|\left(\Delta Z_{k}, \Delta Q_{k}, \Delta U_{k}\right)\right\|^{2} .
\end{aligned}
$$

Set

$$
\left(Z^{k+1}, Q^{k+1}, U^{k+1}\right):=R_{\left(Z^{k}, Q^{k}, U^{k}\right)}\left(\alpha_{k}\left(\Delta Z_{k}, \Delta Q_{k}, \Delta U_{k}\right)\right) .
$$

Copyright (c) by SIAM. Unauthorized reproduction of this article is prohibited. 
Step 4. Replace $k$ by $k+1$ and go to Step 1.

In the following, we have several remarks on the above algorithm.

- In Algorithm 3.1, we set

$$
\left(\Delta Z_{0}, \Delta Q_{0}, \Delta U_{0}\right):=-\operatorname{grad} h\left(Z^{0}, Q^{0}, U^{0}\right) .
$$

- From (3.12), (3.13), and (3.14), we get for all $k \geq 1$,

$$
\left\langle\left(\Delta Z_{k}, \Delta Q_{k}, \Delta U_{k}\right), \operatorname{grad} h\left(Z^{k}, Q^{k}, U^{k}\right)\right\rangle=-\left\|\operatorname{grad} h\left(Z^{k}, Q^{k}, U^{k}\right)\right\|^{2} .
$$

This shows that $\left(\Delta Z_{k}, \Delta Q_{k}, \Delta U_{k}\right)$ is a descent direction of $h$.

- In Algorithm 3.1, we observe that for all $k \geq 1$,

$$
\left\|\Delta Y_{k}\right\| \leq\left\|\left(\Delta Z_{k-1}, \Delta Q_{k-1}, \Delta U_{k-1}\right)\right\| .
$$

- By (3.14) and (3.18), we have that for all $k \geq 1$,

$$
\begin{aligned}
\theta_{k} & \leq \frac{\left\|\operatorname{grad} h\left(Z^{k}, Q^{k}, U^{k}\right)\right\| \cdot\left\|\Delta Y_{k}\right\|}{\left\|\operatorname{grad} h\left(Z^{k-1}, Q^{k-1}, U^{k-1}\right)\right\|^{2}} \\
& \leq \frac{\left\|\operatorname{grad} h\left(Z^{k}, Q^{k}, U^{k}\right)\right\| \cdot\left\|\left(\Delta Z_{k-1}, \Delta Q_{k-1}, \Delta U_{k-1}\right)\right\|}{\left\|\operatorname{grad} h\left(Z^{k-1}, Q^{k-1}, U^{k-1}\right)\right\|^{2}} .
\end{aligned}
$$

- In Step 3 of Algorithm 3.1, the initial step length is set to be $\alpha$. As mentioned in [35], the line-search process may not be very efficient. As in [38], one can derive a reasonable initial step length:

$$
t_{k}=\left|\frac{\left\langle\operatorname{grad} h\left(Z^{k}, Q^{k}, U^{k}\right),\left(\Delta Z_{k}, \Delta Q_{k}, \Delta U_{k}\right)\right\rangle}{\left\|\mathrm{D} H\left(Z^{k}, Q^{k}, U^{k}\right)\left[\left(\Delta Z_{k}, \Delta Q_{k}, \Delta U_{k}\right)\right]\right\|^{2}}\right| .
$$

Sparked by [35], the line-search step (i.e., Step 3) of Algorithm 3.1 can be modified such that if

$$
\left\|\mathrm{D} H\left(Z^{k}, Q^{k}, U^{k}\right)\left[\left(\Delta Z_{k}, \Delta Q_{k}, \Delta U_{k}\right)\right]\right\|>0
$$

and

$$
\begin{aligned}
& h\left(R_{\left(Z^{k}, Q^{k}, U^{k}\right)}\left(t_{k}\left(\Delta Z_{k}, \Delta Q_{k}, \Delta U_{k}\right)\right)\right)-h\left(Z^{k}, Q^{k}, U^{k}\right) \\
\leq & \delta_{1} t_{k}\left\langle\operatorname{grad} h\left(Z^{k}, Q^{k}, U^{k}\right),\left(\Delta Z_{k}, \Delta Q_{k}, \Delta U_{k}\right)\right\rangle-\delta_{2} t_{k}^{2}\left\|\left(\Delta Z_{k}, \Delta Q_{k}, \Delta U_{k}\right)\right\|^{2},
\end{aligned}
$$

then we set $\alpha_{k}=t_{k}$; otherwise, the step length $\alpha_{k}$ can be selected by Step 3 of Algorithm 3.1. The numerical tests in section 6 show that the initial step length (3.19) is very effective.

Finally, we point out that Algorithm 3.1 has some advantages over the constructive methods in $[23,21,32,39,27,28]$. A least squares approach is proposed for solving the general DSIEP, where there is no additional sufficient condition imposed on the prescribed eigenvalues. As shown in section 5, our method can be used to construct a doubly stochastic matrix with both prescribed entries and a spectrum. Even if the DSIEP is not solvable, our method generates a least squares solution.

Copyright (c) by SIAM. Unauthorized reproduction of this article is prohibited. 
4. Global convergence. In this section, we establish the global convergence of Algorithm 3.1. First, we can easily derive the following result. We omit the proof here.

Lemma 4.1. For any given point $\left(Z^{0}, Q^{0}, U^{0}\right) \in \mathcal{O B} \times \mathcal{O}(n) \times \mathcal{U}$, the level set

$$
\Omega:=\left\{(Z, Q, U) \in \mathcal{O B} \times \mathcal{O}(n) \times \mathcal{U} \mid h(Z, Q, U) \leq h\left(Z^{0}, Q^{0}, U^{0}\right)\right\}
$$

is compact.

Remark 4.2. We see that $\mathcal{O B} \times \mathcal{O}(n) \times \mathcal{U}$ is an embedded Riemannian submanifold of $\mathbb{R}^{n \times n} \times \mathbb{R}^{n \times n} \times \mathbb{R}^{n \times n}$. One may rely on the natural inclusion $T_{(Z, Q, U)} \mathcal{O B} \times \mathcal{O}(n) \times$ $\mathcal{U} \subset \mathbb{R}^{n \times n} \times \mathbb{R}^{n \times n} \times \mathbb{R}^{n \times n}$. Thus, the Riemannian gradient grad $h$ defined in (3.8) can be viewed as a continuous nonlinear mapping between $\mathcal{O B} \times \mathcal{O}(n) \times \mathcal{U}$ and $\mathbb{R}^{n \times n} \times$ $\mathbb{R}^{n \times n} \times \mathbb{R}^{n \times n}$. By Lemma 4.1, $\Omega$ is compact. Then there exists a constant $\gamma>0$ such that $[4$, pp. $151-152]$

$$
\|\operatorname{grad} h(Z, Q, U)\| \leq \gamma \quad \forall(Z, Q, U) \in \Omega .
$$

Moreover, for any points $\left(Z_{1}, Q_{1}, U_{1}\right),\left(Z_{2}, Q_{2}, U_{2}\right) \in \mathcal{O B} \times \mathcal{O}(n) \times \mathcal{U}$, the operation $\operatorname{grad} h\left(Z_{2}, Q_{2}, U_{2}\right)-\operatorname{grad} h\left(Z_{1}, Q_{1}, U_{1}\right)$ is meaningful since both $\operatorname{grad} h\left(Z_{1}, Q_{1}, U_{1}\right)$ and $\operatorname{grad} h\left(Z_{2}, Q_{2}, U_{2}\right)$ can be treated as vectors in $\mathbb{R}^{n \times n} \times \mathbb{R}^{n \times n} \times \mathbb{R}^{n \times n}$. By Lemma 4.1, $\Omega$ is compact. Then $\operatorname{grad} h$ is Lipschitz continuous on $\Omega$, i.e., there exists a constant $\beta_{L_{1}}>0$ such that

$\left\|\operatorname{grad} h\left(Z_{2}, Q_{2}, U_{2}\right)-\operatorname{grad} h\left(Z_{1}, Q_{1}, U_{1}\right)\right\| \leq \beta_{L_{1}} \operatorname{dist}\left(\left(Z_{1}, Q_{1}, U_{1}\right),\left(Z_{2}, Q_{2}, U_{2}\right)\right)$

for all $\left(Z_{1}, Q_{1}, U_{1}\right),\left(Z_{2}, Q_{2}, U_{2}\right) \in \Omega$.

LEMMA 4.3. There exists a constant $\nu>0$ such that for all $k$ sufficiently large,

$$
\alpha_{k} \geq \nu \frac{\left\|\operatorname{grad} h\left(Z^{k}, Q^{k}, U^{k}\right)\right\|^{2}}{\left\|\left(\Delta Z_{k}, \Delta Q_{k}, \Delta U_{k}\right)\right\|^{2}} .
$$

Proof. It follows from (3.15), (3.16), and (3.17) that the sequence $\left\{h\left(Z^{k}, Q^{k}, U^{k}\right)\right\}$ is decreasing and bounded below, and is thus convergent. Hence, we have

$\sum_{k=0}^{\infty}\left(-\delta_{1} \alpha_{k}\left\langle\operatorname{grad} h\left(Z^{k}, Q^{k}, U^{k}\right),\left(\Delta Z_{k}, \Delta Q_{k}, \Delta U_{k}\right)\right\rangle+\delta_{2} \alpha_{k}^{2}\left\|\left(\Delta Z_{k}, \Delta Q_{k}, \Delta U_{k}\right)\right\|^{2}\right)<\infty$.

This, together with (3.17), leads to

$$
\sum_{k=0}^{\infty} \alpha_{k}^{2}\left\|\left(\Delta Z_{k}, \Delta Q_{k}, \Delta U_{k}\right)\right\|^{2}<\infty
$$

and

$$
\begin{aligned}
& -\sum_{k=0}^{\infty} \alpha_{k}\left\langle\operatorname{grad} h\left(Z^{k}, Q^{k}, U^{k}\right),\left(\Delta Z_{k}, \Delta Q_{k}, \Delta U_{k}\right)\right\rangle \\
& =\sum_{k=0}^{\infty} \alpha_{k}\left\|\operatorname{grad} h\left(Z^{k}, Q^{k}, U^{k}\right)\right\|^{2}<\infty .
\end{aligned}
$$

Then

$$
\lim _{k \rightarrow \infty} \alpha_{k}\left\|\operatorname{grad} h\left(Z^{k}, Q^{k}, U^{k}\right)\right\|^{2}=0 \quad \text { and } \quad \lim _{k \rightarrow \infty} \alpha_{k}\left\|\left(\Delta Z_{k}, \Delta Q_{k}, \Delta U_{k}\right)\right\|=0 .
$$

Next, we show (4.1). By (3.17), one has, for all $k \geq 1$,

$$
\left\|\operatorname{grad} h\left(Z^{k}, Q^{k}, U^{k}\right)\right\| \leq\left\|\left(\Delta Z_{k}, \Delta Q_{k}, \Delta U_{k}\right)\right\| .
$$

Hence, if $\alpha_{k} \geq 1$ for all $k$, then (4.1) holds for $\nu=1$. 
We now suppose that $\alpha_{k}<1$ for all $k$ sufficiently large. According to Step 3 of Algorithm 3.1, one has, for all $k$ sufficiently large,

$$
\begin{aligned}
& h\left(R_{\left(Z^{k}, Q^{k}, U^{k}\right)}\left(\rho^{-1} \alpha_{k}\left(\Delta Z_{k}, \Delta Q_{k}, \Delta U_{k}\right)\right)\right)-h\left(Z^{k}, Q^{k}, U^{k}\right) \\
& >\delta_{1} \rho^{-1} \alpha_{k}\left\langle\operatorname{grad} h\left(Z^{k}, Q^{k}, U^{k}\right),\left(\Delta Z_{k}, \Delta Q_{k}, \Delta U_{k}\right)\right\rangle \\
& \quad-\delta_{2} \rho^{-2} \alpha_{k}^{2}\left\|\left(\Delta Z_{k}, \Delta Q_{k}, \Delta U_{k}\right)\right\|^{2} .
\end{aligned}
$$

We note that the pullback function $\widehat{h}$ is continuously differentiable, since both the cost function $h$ and the retraction mapping $R$ are continuously differentiable. Then there exist two constants $\kappa>0$ and $\beta_{L_{2}}>0$ such that

$$
\left\|\operatorname{grad} \widehat{h}_{X}\left(\eta_{X}\right)-\operatorname{grad} \widehat{h}_{X}\left(\xi_{X}\right)\right\| \leq \beta_{L_{2}}\left\|\eta_{X}-\xi_{X}\right\|
$$

for any $X \in \Omega$ and $\xi_{X}, \eta_{X} \in T_{X} \mathcal{O B} \times \mathcal{O}(n) \times \mathcal{U}$ with $\left\|\eta_{X}\right\|,\left\|\xi_{X}\right\| \leq \kappa$. Also, we have by (4.3), for all $k$ sufficiently large,

$$
\alpha_{k}\left\|\left(\Delta Z_{k}, \Delta Q_{k}, \Delta U_{k}\right)\right\| \leq \kappa .
$$

By the mean-value theorem, (3.11), (3.17), (4.5), and (4.6), there is a $\omega_{k} \in(0,1)$ such that for all $k$ sufficiently large,

$$
\begin{aligned}
& h\left(R_{\left(Z^{k}, Q^{k}, U^{k}\right)}\left(\rho^{-1} \alpha_{k}\left(\Delta Z_{k}, \Delta Q_{k}, \Delta U_{k}\right)\right)\right)-h\left(Z^{k}, Q^{k}, U^{k}\right) \\
= & h\left(R_{\left(Z^{k}, Q^{k}, U^{k}\right)}\left(\rho^{-1} \alpha_{k}\left(\Delta Z_{k}, \Delta Q_{k}, \Delta U_{k}\right)\right)\right)-h\left(R_{\left(Z^{k}, Q^{k}, U^{k}\right)}\left(0_{\left(Z^{k}, Q^{k}, U^{k}\right)}\right)\right) \\
= & \widehat{h}_{\left(Z^{k}, Q^{k}, U^{k}\right)}\left(\rho^{-1} \alpha_{k}\left(\Delta Z_{k}, \Delta Q_{k}, \Delta U_{k}\right)\right)-\widehat{h}_{\left(Z^{k}, Q^{k}, U^{k}\right)}\left(0_{\left(Z^{k}, Q^{k}, U^{k}\right)}\right) \\
= & \rho^{-1} \alpha_{k}\left\langle\operatorname{grad} \widehat{h}_{\left(Z^{k}, Q^{k}, U^{k}\right)}\left(\omega_{k} \rho^{-1} \alpha_{k}\left(\Delta Z_{k}, \Delta Q_{k}, \Delta U_{k}\right)\right),\left(\Delta Z_{k}, \Delta Q_{k}, \Delta U_{k}\right)\right\rangle \\
(4.7)= & \rho^{-1} \alpha_{k}\left\langle\operatorname{grad} \widehat{h}_{\left(Z^{k}, Q^{k}, U^{k}\right)}\left(0_{\left(Z^{k}, Q^{k}, U^{k}\right)}\right),\left(\Delta Z_{k}, \Delta Q_{k}, \Delta U_{k}\right)\right\rangle \\
& +\rho^{-1} \alpha_{k}\left\langle\left(\Delta Z_{k}, \Delta Q_{k}, \Delta U_{k}\right), \operatorname{grad} \widehat{h}_{\left(Z^{k}, Q^{k}, U^{k}\right)}\left(\omega_{k} \rho^{-1} \alpha_{k}\left(\Delta Z_{k}, \Delta Q_{k}, \Delta U_{k}\right)\right)\right\rangle \\
& -\rho^{-1} \alpha_{k}\left\langle\left(\Delta Z_{k}, \Delta Q_{k}, \Delta U_{k}\right), \operatorname{grad} \widehat{h}_{\left(Z^{k}, Q^{k}, U^{k}\right)}\left(0_{\left(Z^{k}, Q^{k}, U^{k}\right)}\right)\right\rangle \\
\leq & \rho^{-1} \alpha_{k}\left\langle\operatorname{grad} \widehat{h}_{\left(Z^{k}, Q^{k}, U^{k}\right)}\left(0_{\left(Z^{k}, Q^{k}, U^{k}\right)}\right),\left(\Delta Z_{k}, \Delta Q_{k}, \Delta U_{k}\right)\right\rangle \\
& +\beta_{L_{2}} \omega_{k} \rho^{-2} \alpha_{k}^{2}\left\|\left(\Delta Z_{k}, \Delta Q_{k}, \Delta U_{k}\right)\right\|^{2} \\
\leq & -\rho^{-1} \alpha_{k}\left\|\operatorname{grad} h\left(Z^{k}, Q^{k}, U^{k}\right)\right\|^{2}+\beta_{L_{2}} \rho^{-2} \alpha_{k}^{2}\left\|\left(\Delta Z_{k}, \Delta Q_{k}, \Delta U_{k}\right)\right\|^{2} .
\end{aligned}
$$

Combining (3.17) and (4.4) with (4.7), we have for all $k$ sufficiently large,

$$
\alpha_{k}>\frac{\left(1-\delta_{1}\right) \rho\left\|\operatorname{grad} h\left(Z^{k}, Q^{k}, U^{k}\right)\right\|^{2}}{\left(\delta_{2}+\beta_{L_{2}}\right)\left\|\left(\Delta Z_{k}, \Delta Q_{k}, \Delta U_{k}\right)\right\|^{2}}
$$

Then we get (4.1) by setting $\nu=\min \left\{1,\left(\left(1-\delta_{1}\right) \rho\right) /\left(\delta_{2}+\beta_{L_{2}}\right)\right\}$.

By (4.1) and (4.2), we have the following Riemannian analogy of the Zoutendijk condition (see [26] for more details).

Lemma 4.4. One has

$$
\sum_{k=0}^{\infty} \frac{\left\|\operatorname{grad} h\left(Z^{k}, Q^{k}, U^{k}\right)\right\|^{4}}{\left\|\left(\Delta Z_{k}, \Delta Q_{k}, \Delta U_{k}\right)\right\|^{2}}<\infty
$$

We now establish the global convergence of Algorithm 3.1. The proof can be viewed as a generalization of [36, Theorem 3.3].

Copyright $($ C by SIAM. Unauthorized reproduction of this article is prohibited. 
Theorem 4.5. Let $\left\{\left(Z^{k}, Q^{k}, U^{k}\right)\right\}$ be the sequence generated by Algorithm 3.1. Then we have

$$
\liminf _{k \rightarrow \infty}\left\|\operatorname{grad} h\left(Z^{k}, Q^{k}, U^{k}\right)\right\|=0 .
$$

Proof. For the sake of contradiction, we assume that there exists a constant $\epsilon>0$ such that

$$
\left\|\operatorname{grad} h\left(Z^{k}, Q^{k}, U^{k}\right)\right\| \geq \epsilon \quad \forall k .
$$

We have by (3.12), (3.13), (3.14), and (3.18), for all $k \geq 1$,

$$
\begin{aligned}
& \left\|\left(\Delta Z_{k}, \Delta Q_{k}, \Delta U_{k}\right)\right\|^{2} \\
& =\beta_{k}^{2}\left\|\Delta Y_{k}\right\|^{2}-\left(\theta_{k}^{2}-1\right)\left\|\operatorname{grad} h\left(Z^{k}, Q^{k}, U^{k}\right)\right\|^{2} \\
& \leq \beta_{k}^{2}\left\|\left(\Delta Z_{k-1}, \Delta Q_{k-1}, \Delta U_{k-1}\right)\right\|^{2}-\left(\theta_{k}^{2}-1\right)\left\|\operatorname{grad} h\left(Z^{k}, Q^{k}, U^{k}\right)\right\|^{2} .
\end{aligned}
$$

We get by (3.13), (4.9), and (4.10),

$$
\begin{aligned}
& \frac{\left.\| \Delta Z_{k}, \Delta Q_{k}, \Delta U_{k}\right) \|^{2}}{\left\|\operatorname{grad} h\left(Z^{k}, Q^{k}, U^{k}\right)\right\|^{4}} \\
& \leq \frac{\beta_{k}^{2}\left\|\left(\Delta Z_{k-1}, \Delta Q_{k-1}, \Delta U_{k-1}\right)\right\|^{2}}{\left\|\operatorname{grad} h\left(Z^{k}, Q^{k}, U^{k}\right)\right\|^{4}}-\frac{\theta_{k}^{2}-1}{\left\|\operatorname{grad} h\left(Z^{k}, Q^{k}, U^{k}\right)\right\|^{2}} \\
& \leq \frac{\left\|\left(\Delta Z_{k-1}, \Delta Q_{k-1}, \Delta U_{k-1}\right)\right\|^{2}}{\left\|\operatorname{grad} h\left(Z^{k-1}, Q^{k-1}, U^{k-1}\right)\right\|^{4}}-\frac{\theta_{k}^{2}}{\left\|\operatorname{grad} h\left(Z^{k}, Q^{k}, U^{k}\right)\right\|^{2}}+\frac{1}{\left\|\operatorname{grad} h\left(Z^{k}, Q^{k}, U^{k}\right)\right\|^{2}} \\
& \leq \frac{\left\|\left(\Delta Z_{k-1}, \Delta Q_{k-1}, \Delta U_{k-1}\right)\right\|^{2}}{\left\|\operatorname{grad} h\left(Z^{k-1}, Q^{k-1}, U^{k-1}\right)\right\|^{4}}+\frac{1}{\left\|\operatorname{grad} h\left(Z^{k}, Q^{k}, U^{k}\right)\right\|^{2}} \\
& \leq \sum_{j=0}^{k} \frac{1}{\left\|\operatorname{grad} h\left(Z^{j}, Q^{j}, U^{j}\right)\right\|^{2}} \leq \frac{k+1}{\epsilon^{2}},
\end{aligned}
$$

where the last inequality implies

$$
\sum_{k=0}^{\infty} \frac{\left\|\operatorname{grad} h\left(Z^{k}, Q^{k}, U^{k}\right)\right\|^{4}}{\left\|\left(\Delta Z_{k}, \Delta Q_{k}, \Delta U_{k}\right)\right\|^{2}} \geq \epsilon^{2} \sum_{k=0}^{\infty} \frac{1}{k+1}=\infty .
$$

This contradicts the Riemannian Zoutendijk condition (4.8). This completes the proof.

5. DSIEP with prescribed entries. In this section, we consider the DSIEP with prescribed entries (DSIEP-PE). In many applications, the underlying structure of a desired stochastic matrix is often characterized by the prescribed entries at arbitrary locations. The DSIEP-PE can be stated as follows.

DSIEP-PE. Given a self-conjugate set of complex numbers $\left\{\lambda_{1}, \lambda_{2}, \ldots, \lambda_{n}\right\}$, find a doubly stochastic matrix $C \in \mathbb{R}^{n \times n}$ such that its eigenvalues are $\lambda_{1}, \lambda_{2}, \ldots, \lambda_{n}$ exactly and

$$
C_{i j}=\left(C_{a}\right)_{i j} \forall(i, j) \in \mathcal{L},
$$

where $C_{a} \in \mathbb{R}^{n \times n}$ is a prescribed nonnegative matrix and $\mathcal{L} \subset \mathcal{N}$ is a given index subset.

Copyright $@$ by SIAM. Unauthorized reproduction of this article is prohibited. 
As in section 3, let $\Lambda \in \mathbb{R}^{n \times n}$ be a block diagonal matrix with the given eigenvalues $\left\{\lambda_{1}, \lambda_{2}, \ldots, \lambda_{n}\right\}$. Define the matrix $\widehat{G} \in \mathbb{R}^{n \times n}$ by

$$
\widehat{G}_{i j}:= \begin{cases}1, & \text { if }(i, j) \in \mathcal{L}, \\ 0, & \text { otherwise }\end{cases}
$$

Then we define a nonnegative matrix $\widehat{C}_{a}$ and a diagonal matrix $\widehat{I}_{n}$ by

$$
\widehat{C}_{a}:=\widehat{G} \odot C_{a} \text { and } \widehat{I}_{n}:=I_{n}-\operatorname{Diag}\left(\widehat{C}_{a} \mathbf{e}\right),
$$

where $\operatorname{Diag}(\mathbf{a})$ is a diagonal matrix with the vector $\mathbf{a}$ as the diagonal. In addition, we assume that the given index subset $\mathcal{L}$ is such that $\sum_{j=1}^{n}\left(\widehat{C}_{a}\right)_{i j}<1$ for $i=1, \ldots, n$. In this case, the diagonal matrix $\widehat{I}_{n}$ is nonsingular. Thus, the DSIEP-PE aims to solve the nonlinear matrix equation

$$
\Phi(Z, Q, U):=\left(\Phi_{1}(Z, Q, U), \Phi_{2}(Z)\right)=0
$$

for $(Z, Q, U) \in \widehat{\mathcal{O B}} \times \mathcal{O}(n) \times \mathcal{U}$, where the set $\widehat{\mathcal{O B}}$ is a manifold defined by

$$
\widehat{\mathcal{O B}}:=\left\{Z \in \mathbb{R}^{n \times n} \mid \operatorname{diag}\left(Z Z^{T}\right)=\widehat{I}_{n}, \widehat{G} \odot Z=0\right\},
$$

and the mappings $\Phi_{1}: \widehat{\mathcal{O B}} \times \mathcal{O}(n) \times \mathcal{U} \rightarrow \mathbb{R}^{n \times n}$ and $\Phi_{2}: \widehat{\mathcal{O B}} \rightarrow \mathbb{R}^{n}$ are defined by

$$
\begin{cases}\Phi_{1}(Z, Q, U):=\widehat{C}_{a}+Z \odot Z-Q(\Lambda+U) Q^{T}, & (Z, Q, U) \in \widehat{\mathcal{O B}} \times \mathcal{O}(n) \times \mathcal{U}, \\ \Phi_{2}(Z):=\left(\widehat{C}_{a}+Z \odot Z\right)^{T} \mathbf{e}-\mathbf{e}, & Z \in \widehat{\mathcal{O B}}\end{cases}
$$

Notice that the dimension of $\widehat{\mathcal{O B}} \times \mathcal{O}(n) \times \mathcal{U}$ is given by

$$
\operatorname{dim}(\widehat{\mathcal{O B}} \times \mathcal{O}(n) \times \mathcal{U})=n(n-1)-|\mathcal{L}|+\frac{n(n-1)}{2}+|\mathcal{J}| .
$$

We see that the nonlinear equation $\Phi(Z, Q, U)=0$ is an underdetermined system defined from the product manifold $\widehat{\mathcal{O B}} \times \mathcal{O}(n) \times \mathcal{U}$ to the Euclidean space $\mathbb{R}^{n \times n} \times \mathbb{R}^{n}$ if $n$ is large and the number $|\mathcal{L}|$ of prescribed entries is not large.

If we find a solution $(\bar{Z}, \bar{Q}, \bar{U}) \in \widehat{\mathcal{O B}} \times \mathcal{O}(n) \times \mathcal{U}$ to $\Phi(Z, Q, U)=0$, then $\bar{C}=$ $\widehat{G} \odot C_{a}+\bar{Z} \odot \bar{Z}$ is a solution to the DSIEP-PE. Alternatively, one may solve the DSIEP$\mathrm{PE}$ by finding a global solution to the following nonlinear least squares problem:

$$
\begin{array}{ll}
\min & \phi(Z, Q, U):=\frac{1}{2}\|\Phi(Z, Q, U)\|_{F}^{2} \\
\text { s.t. } & Z \in \widehat{\mathcal{O B}}, \quad Q \in \mathcal{O}(n), \quad U \in \mathcal{U} .
\end{array}
$$

Let $\widehat{\mathcal{O B}} \times \mathcal{O}(n) \times \mathcal{U}$ be equipped with the Riemannian metric defined as in (3.3). It follows that the tangent space of $\widehat{\mathcal{O B}}$ at a point $Z \in \widehat{\mathcal{O B}}$ is given by

$$
T_{Z} \widehat{\mathcal{O B}}=\left\{W \in \mathbb{R}^{n \times n} \mid \operatorname{diag}\left(Z W^{T}\right)=0, \widehat{G} \odot W=0\right\} .
$$

Then the orthogonal projection of a point $\xi \in \mathbb{R}^{n \times n}$ onto $T_{Z} \widehat{\mathcal{O B}}$ is given by

$$
\widehat{\Pi}_{Z} \xi=(E-\widehat{G}) \odot \xi-\widehat{I}_{n}^{-1} \operatorname{diag}\left(Z((E-\widehat{G}) \odot \xi)^{T}\right) Z,
$$

Copyright (c) by SIAM. Unauthorized reproduction of this article is prohibited. 
where $E$ is an $n$-by- $n$ matrix of ones. The retraction on $\widehat{\mathcal{O B}}$ at a point $Z \in \widehat{\mathcal{O B}}$ can be defined by

$$
\widehat{R}_{Z}\left(\xi_{Z}\right)=\widehat{I}_{n}^{\frac{1}{2}}\left(\operatorname{diag}\left(\left(Z+\xi_{Z}\right)\left(Z+\xi_{Z}\right)^{T}\right)\right)^{-\frac{1}{2}}\left(Z+\xi_{Z}\right) \quad \forall \xi_{Z} \in T_{Z} \widehat{\mathcal{O B}}
$$

The vector transport on $\widehat{\mathcal{O B}}$ is given by

$\widehat{\mathcal{T}}_{\eta_{Z}}\left(\xi_{Z}\right):=\Pi_{\widehat{R}_{Z}\left(\eta_{Z}\right)}\left(\xi_{Z}\right)=\xi_{Z}-\left(\operatorname{diag}\left(\left(Z+\eta_{Z}\right)\left(Z+\eta_{Z}\right)^{T}\right)\right)^{-1} \operatorname{diag}\left(\left(Z+\eta_{Z}\right) \xi_{Z}^{T}\right)\left(Z+\eta_{Z}\right)$

for any $Z \in \widehat{\mathcal{O B}}$ and $\xi_{Z}, \eta_{Z} \in T_{Z} \widehat{\mathcal{O B}}$.

Next, we establish explicit formulas for the differential of the smooth mapping $\Phi$ defined in (5.1) and the Riemannian gradient of the cost function $\phi$ defined in Problem (5.2). As in section 3, the differential

$$
\mathrm{D} \Phi(Z, Q, U): T_{(Z, Q, U)} \widehat{\mathcal{O B}} \times \mathcal{O}(n) \times \mathcal{U} \rightarrow T_{\Phi(Z, Q, U)} \mathbb{R}^{n \times n} \times \mathbb{R}^{n} \simeq \mathbb{R}^{n \times n} \times \mathbb{R}^{n}
$$

of $\Phi$ at a point $(Z, Q, U) \in \widehat{\mathcal{O B}} \times \mathcal{O}(n) \times \mathcal{U}$ is determined by

$$
\begin{aligned}
& \mathrm{D} \Phi(Z, Q, U)[(\Delta Z, \Delta Q, \Delta U)]=\left(\mathrm{D}_{1}(Z, Q, U)[(\Delta Z, \Delta Q, \Delta U)], \mathrm{D} \Phi_{2}(Z)[\Delta Z]\right) \\
= & \left(2 Z \odot \Delta Z+\left[Q(\Lambda+U) Q^{T}, \Delta Q Q^{T}\right]-Q \Delta U Q^{T}, 2(Z \odot \Delta Z)^{T} \mathbf{e}\right)
\end{aligned}
$$

for all $(\Delta Z, \Delta Q, \Delta U) \in T_{(Z, Q, U)} \widehat{\mathcal{O B}} \times \mathcal{O}(n) \times \mathcal{U}$. The adjoint

$$
(\mathrm{D} \Phi(Z, Q, U))^{*}: T_{\Phi(Z, Q, U)} \mathbb{R}^{n \times n} \times \mathbb{R}^{n} \rightarrow T_{(Z, Q, U)} \widehat{\mathcal{O B}} \times \mathcal{O}(n) \times \mathcal{U}
$$

of $\mathrm{D} \Phi(Z, Q, U)$ is determined by

$$
(\mathrm{D} \Phi(Z, Q, U))^{*}[\Delta W]=\left((\mathrm{D} \Phi)_{1}^{*}[\Delta W],(\mathrm{D} \Phi)_{2}^{*}[\Delta W],(\mathrm{D} \Phi)_{3}^{*}[\Delta W]\right)
$$

for all

$$
\Delta W:=\left(\Delta W_{1}, \Delta W_{2}\right) \in T_{\Phi(Z, Q, U)} \mathbb{R}^{n \times n} \times \mathbb{R}^{n},
$$

where for each $\Delta W:=\left(\Delta W_{1}, \Delta W_{2}\right) \in T_{\Phi(Z, Q, U)} \mathbb{R}^{n \times n} \times \mathbb{R}^{n}$,

$$
\left\{\begin{aligned}
(\mathrm{D} \Phi)_{1}^{*}[\Delta W]= & 2 Z \odot\left(\Delta W_{1}+\mathbf{e}\left(\Delta W_{2}\right)^{T}\right) \\
& -2 \widehat{I}_{n}^{-1} \operatorname{diag}\left(Z\left(Z \odot\left(\Delta W_{1}+\mathbf{e}\left(\Delta W_{2}\right)^{T}\right)\right)^{T}\right) Z, \\
(\mathrm{D} \Phi)_{2}^{*}[\Delta W]= & \frac{1}{2}\left(\left[Q(\Lambda+U) Q^{T},\left(\Delta W_{1}\right)^{T}\right]+\left[Q(\Lambda+U)^{T} Q^{T}, \Delta W_{1}\right]\right) Q, \\
(\mathrm{D} \Phi)_{3}^{*}[\Delta W]= & -G \odot\left(Q^{T} \Delta W_{1} Q\right) .
\end{aligned}\right.
$$

Then the Riemannian gradient of $\phi$ at a point $(Z, Q, U) \in \widehat{\mathcal{O B}} \times \mathcal{O}(n) \times \mathcal{U}$ is given by $[4$, p. 184]

$$
\operatorname{grad} \phi(Z, Q, U)=(\mathrm{D} \Phi(Z, Q, U))^{*}[\Phi(Z, Q, U)],
$$

where $(\mathrm{D} \Phi(Z, Q, U))^{*}[\cdot]$ is given by $(5.3)$.

Similarly, a Riemannian gradient flow method for the cost function $\phi$ over the product manifold $\widehat{\mathcal{O B}} \times \mathcal{O}(n) \times \mathcal{U}$ is given by

$$
\frac{d(Z, Q, U)}{d t}=-\operatorname{grad} \phi(Z, Q, U)
$$

Copyright $@$ by SIAM. Unauthorized reproduction of this article is prohibited. 
which is an initial value problem if given an initial value $(Z(0), Q(0), U(0)) \in \widehat{\mathcal{O B}} \times$ $\mathcal{O}(n) \times \mathcal{U}$. Then one may get a solution to the DSIEP-PE (5.2) by using existing ODE solvers for the above differential equation.

As in section 3.3, one may develop a Riemannian Fletcher-Reeves conjugate gradient algorithm for solving the DSIEP-PE, i.e., one may solve the DSIEP-PE (5.2) by using Algorithm 3.1. The corresponding global convergence can also be established.

6. Numerical tests. In this section, we report the numerical performance of Algorithm 3.1 for solving the DSIEP (3.2) and the DSIEP-PE (5.2). All numerical tests are carried out by using MATLAB 7.1 running on a workstation with an Intel Xeon CPU E5-2687W at $3.10 \mathrm{GHz}$ and $32 \mathrm{~GB}$ of RAM. To generate a random doubly stochastic matrix, we may use Birkhoff's theorem [7], which says that any $n$-by- $n$ doubly stochastic matrix $A$ can be written as a convex combination of $m$ permutation matrices for $m \leq(n-1)^{2}+1$.

For Algorithm 3.1, the starting points are generated randomly by the built-in functions rand and schur: for the DSIEP,

$$
\left\{\begin{array}{l}
\widehat{Z} \odot \widehat{Z}=\operatorname{rand}(n, n), Z^{0}=\left(\operatorname{diag}\left(\widehat{Z} \widehat{Z}^{T}\right)\right)^{-\frac{1}{2}} \widehat{Z}=Z(0) \in \mathcal{O B}, \\
{\left[Q^{0}, \widehat{U}\right]=\operatorname{schur}\left(Z^{0} \odot Z^{0},{ }^{\prime} \text { real }{ }^{\prime}\right)=[Q(0), \widehat{U}], \quad U^{0}=G \odot \widehat{U}=U(0),}
\end{array}\right.
$$

and for the DSIEP-PE,

$$
\left\{\begin{array}{l}
\widehat{Z} \odot \widehat{Z}=\operatorname{rand}(n, n), Z^{0}=\widehat{I}_{n}^{\frac{1}{2}}\left(\operatorname{diag}\left((\widehat{W} \odot \widehat{Z})(\widehat{W} \odot \widehat{Z})^{T}\right)\right)^{-\frac{1}{2}}(\widehat{W} \odot \widehat{Z})=Z(0) \in \widehat{\mathcal{O B}}, \\
{\left[Q^{0}, \widehat{U}\right]=\operatorname{schur}\left(\widehat{C}_{a}+Z^{0} \odot Z^{0},{ }^{\prime} \operatorname{real}^{\prime}\right)=[Q(0), \widehat{U}], \quad U^{0}=G \odot \widehat{U}=U(0),}
\end{array}\right.
$$

where $\widehat{W}:=E-\widehat{G}$, with $E$ being an $n$-by- $n$ matrix of all ones.

In our numerical tests, the stopping criterion for Algorithm 3.1 is set to be

$$
\left\|H\left(Z^{k}, Q^{k}, U^{k}\right)\right\|_{F} \leq 10^{-12} \text { or } \quad\left\|\Phi\left(Z^{k}, Q^{k}, U^{k}\right)\right\|_{F} \leq 10^{-12} .
$$

As in [36], we also set $\alpha=1.4, \rho=0.5, \delta_{1}=10^{-3}$, and $\delta_{2}=10^{-8}$.

In our tests, "CT.", "IT.", "NF.", "Err.", and "Res." mean the total computing time, the number of iterations, the number of function evaluations, the error $\left\|H\left(Z^{k}, Q^{k}, U^{k}\right)\right\|_{F}$ or $\left\|\Phi\left(Z^{k}, Q^{k}, U^{k}\right)\right\|_{F}$, and the residual $\left\|\operatorname{grad} h\left(Z^{k}, Q^{k}, U^{k}\right)\right\|$ or $\left\|\operatorname{grad} \phi\left(Z^{k}, Q^{k}, U^{k}\right)\right\|$ at the final iterate of the corresponding algorithm accordingly.

We consider two examples with different problem sizes $n$.

Example 6.1. We consider the DSIEP with varying $n$. Let $\widetilde{C}=\sum_{j=1}^{n} c_{j} P_{j}$ be a random $n \times n$ doubly stochastic matrix where $\left\{c_{j} \geq 0\right\}$ are generated randomly such that $\sum_{j=1}^{n} c_{j}=1$ and $\left\{P_{j}\right\}$ are $n$ random permutation matrices. We choose the eigenvalues of $\widehat{C}$ as the prescribed spectra.

Example 6.2. We consider the DSIEP-PE with varying $n$. Let $\widetilde{C}=\sum_{j=1}^{n} c_{j} P_{j}$ be a random $n \times n$ doubly stochastic matrix where $\left\{c_{j} \geq 0\right\}$ are generated randomly such that $\sum_{j=1}^{n} c_{j}=1$ and $\left\{P_{j}\right\}$ are $n$ random permutation matrices. We choose the eigenvalues of $\widehat{C}$ as the prescribed spectra. Also, we choose the index subset $\mathcal{L}:=\left\{(i, j) \mid 0.02 \leq(\widehat{C})_{i j} \leq 0.03, i, j=1, \ldots, n\right\}$. The prescribed nonnegative matrix $C_{a} \in \mathbb{R}^{n \times n}$ is such that $\left(C_{a}\right)_{i j}=\widehat{C}_{i j}$ if $(i, j) \in \mathcal{L}$ and $\left(C_{a}\right)_{i j}=0$ otherwise.

For demonstration purposes, in Tables 1 and 2 we report the numerical results for Examples 6.1 and 6.2 with different problem sizes $n$, where the initial step length guess (3.19) may be used in Algorithm 3.1. 
TABLE 1

Numerical results for Example 6.1.

\begin{tabular}{|c|r|r|r|r|l|l|}
\hline Alg. & $n$ & CT. & IT. & NF. & Err. & Res. \\
\hline & 100 & $2.2 \mathrm{~s}$ & 742 & 747 & $9.6 \times 10^{-13}$ & $1.6 \times 10^{-13}$ \\
& 200 & $14 \mathrm{~s}$ & 1454 & 1460 & $9.9 \times 10^{-13}$ & $1.2 \times 10^{-13}$ \\
Alg. & 500 & $02 \mathrm{~m} 22 \mathrm{~s}$ & 1548 & 1564 & $9.8 \times 10^{-13}$ & $1.3 \times 10^{-13}$ \\
3.1 & 800 & $23 \mathrm{~m} 17 \mathrm{~s}$ & 4848 & 4855 & $9.9 \times 10^{-13}$ & $9.5 \times 10^{-14}$ \\
& 1000 & $46 \mathrm{~m} 13 \mathrm{~s}$ & 5327 & 5334 & $9.9 \times 10^{-13}$ & $1.3 \times 10^{-13}$ \\
& 1500 & $03 \mathrm{~h} 46 \mathrm{~m} 13 \mathrm{~s}$ & 3820 & 21211 & $9.9 \times 10^{-13}$ & $6.3 \times 10^{-13}$ \\
& 2000 & $07 \mathrm{~h} 47 \mathrm{~m} 51 \mathrm{~s}$ & 8507 & 9110 & $9.9 \times 10^{-13}$ & $7.5 \times 10^{-13}$ \\
& 3000 & $\geq 15 \mathrm{~h}$ & $*$ & $*$ & $*$ & $*$ \\
\hline & 100 & $1.0 \mathrm{~s}$ & 278 & 281 & $9.8 \times 10^{-13}$ & $5.7 \times 10^{-13}$ \\
Alg. & 200 & $4.4 \mathrm{~s}$ & 346 & 349 & $8.6 \times 10^{-13}$ & $5.8 \times 10^{-13}$ \\
3.1 & 500 & $01 \mathrm{~m} \mathrm{00} \mathrm{s}$ & 419 & 422 & $9.3 \times 10^{-13}$ & $5.4 \times 10^{-13}$ \\
with & 800 & $03 \mathrm{~m} \mathrm{36} \mathrm{s}$ & 461 & 464 & $9.3 \times 10^{-13}$ & $3.5 \times 10^{-13}$ \\
$(3.19)$ & 1000 & $06 \mathrm{~m} 44 \mathrm{~s}$ & 477 & 480 & $9.3 \times 10^{-13}$ & $5.3 \times 10^{-13}$ \\
& 1500 & $20 \mathrm{~m} \mathrm{50} \mathrm{s}$ & 521 & 524 & $9.8 \times 10^{-13}$ & $7.4 \times 10^{-13}$ \\
& 2000 & $58 \mathrm{~m} \mathrm{37} \mathrm{s}$ & 558 & 561 & $9.8 \times 10^{-13}$ & $5.1 \times 10^{-13}$ \\
& 3000 & $03 \mathrm{~h} 17 \mathrm{~m} 12 \mathrm{~s}$ & 629 & 632 & $9.9 \times 10^{-13}$ & $7.6 \times 10^{-13}$ \\
\hline
\end{tabular}

TABLE 2

Numerical results for Example 6.2.

\begin{tabular}{|c|r|r|c|c|l|l|}
\hline Alg. & $n$ & CT. & IT. & NF. & Err. & Res. \\
\hline & 100 & $3.9 \mathrm{~s}$ & 855 & 2521 & $9.8 \times 10^{-13}$ & $4.0 \times 10^{-13}$ \\
& 200 & $9.8 \mathrm{~s}$ & 996 & 1001 & $9.8 \times 10^{-13}$ & $1.4 \times 10^{-13}$ \\
Alg. & 500 & $05 \mathrm{~m} 45 \mathrm{~s}$ & 1817 & 7929 & $9.9 \times 10^{-13}$ & $4.4 \times 10^{-13}$ \\
3.1 & 800 & $21 \mathrm{~m} 48 \mathrm{~s}$ & 4694 & 4701 & $9.9 \times 10^{-13}$ & $9.1 \times 10^{-13}$ \\
& 1000 & $39 \mathrm{~m} 57 \mathrm{~s}$ & 5152 & 5159 & $9.9 \times 10^{-13}$ & $1.3 \times 10^{-13}$ \\
& 1500 & $02 \mathrm{~h} 19 \mathrm{~m} 36 \mathrm{~s}$ & 2736 & 13136 & $9.9 \times 10^{-13}$ & $6.4 \times 10^{-13}$ \\
& 2000 & $07 \mathrm{~h} 32 \mathrm{~m} \mathrm{05} \mathrm{s}$ & 8966 & 9713 & $9.9 \times 10^{-13}$ & $3.8 \times 10^{-13}$ \\
& 3000 & $\geq 15 \mathrm{~h}$ & $*$ & $*$ & $*$ & $*$ \\
\hline & 100 & $1.3 \mathrm{~s}$ & 397 & 400 & $9.3 \times 10^{-13}$ & $4.3 \times 10^{-13}$ \\
Alg. & 200 & $36 \mathrm{~s}$ & 406 & 409 & $9.4 \times 10^{-13}$ & $5.2 \times 10^{-13}$ \\
3.1 & 500 & $48 \mathrm{~s}$ & 431 & 434 & $9.9 \times 10^{-13}$ & $1.8 \times 10^{-13}$ \\
with & 800 & $02 \mathrm{~m} \mathrm{34} \mathrm{s}$ & 451 & 454 & $9.3 \times 10^{-13}$ & $3.8 \times 10^{-13}$ \\
$(3.19)$ & 1000 & $04 \mathrm{~m} \mathrm{36} \mathrm{s}$ & 480 & 483 & $9.8 \times 10^{-13}$ & $3.6 \times 10^{-13}$ \\
& 1500 & $14 \mathrm{~m} 46 \mathrm{~s}$ & 520 & 523 & $9.8 \times 10^{-13}$ & $5.8 \times 10^{-13}$ \\
& 2000 & $35 \mathrm{~m} 53 \mathrm{~s}$ & 562 & 565 & $9.7 \times 10^{-13}$ & $5.4 \times 10^{-13}$ \\
& 3000 & $02 \mathrm{~h} 02 \mathrm{~m} \mathrm{58 \textrm {s }}$ & 630 & 633 & $9.9 \times 10^{-13}$ & $6.0 \times 10^{-13}$ \\
\hline
\end{tabular}

We see from Tables 1 and 2 that Algorithm 3.1 is very efficient for solving largescale problems. Moreover, the initial step length guess (3.19) substantially reduces the number of iterations and thus improves the efficiency.

Finally, to illustrate the efficiency of our algorithm, we compare Algorithm 3.1 with the ODE solver ode113 provided by MATLAB for solving the differential equations (3.9) and (5.4) (as in [10]). For Algorithm 3.1 and the ODE solver ode113 for solving (3.9) and (5.4), the starting points are randomly generated as in (6.1). For comparison purposes, in our numerical tests, the stopping criteria for Algorithm 3.1 and the ODE solver ode113 for solving (3.9) and (5.4) are, respectively, set to be

$$
\left\|H\left(Z^{k}, Q^{k}, U^{k}\right)\right\|_{F} \leq 10^{-8} \text { and }\left\|\Phi\left(Z^{k}, Q^{k}, U^{k}\right)\right\|_{F} \leq 10^{-8} .
$$


TABLE 3

Comparison of Algorithm 3.1 and ode113 for Example 6.1.

\begin{tabular}{|c|r|r|r|r|l|l|}
\hline Alg. & $n$ & CT. & IT. & \multicolumn{1}{c|}{ NF. } & Err. & Res. \\
\hline & 10 & $0.98 \mathrm{~s}$ & 2034 & 4336 & $8.48 \times 10^{-9}$ & $1.63 \times 10^{-9}$ \\
& 20 & $1.09 \mathrm{~s}$ & 1989 & 4304 & $7.44 \times 10^{-9}$ & $1.29 \times 10^{-9}$ \\
ode113 & 50 & $4.35 \mathrm{~s}$ & 3373 & 7376 & $9.98 \times 10^{-9}$ & $1.26 \times 10^{-9}$ \\
for & 80 & $11.0 \mathrm{~s}$ & 4596 & 10138 & $9.32 \times 10^{-9}$ & $9.71 \times 10^{-10}$ \\
$(3.9)$ & 100 & $19.6 \mathrm{~s}$ & 5667 & 12520 & $9.31 \times 10^{-9}$ & $8.97 \times 10^{-10}$ \\
& 150 & $59.0 \mathrm{~s}$ & 7520 & 16760 & $9.64 \times 10^{-9}$ & $7.52 \times 10^{-10}$ \\
& 200 & $02 \mathrm{~m} 15 \mathrm{~s}$ & 9812 & 21929 & $9.87 \times 10^{-9}$ & $6.85 \times 10^{-10}$ \\
& 300 & $09 \mathrm{~m} 26 \mathrm{~s}$ & 13518 & 30332 & $9.92 \times 10^{-9}$ & $5.53 \times 10^{-10}$ \\
& 400 & $26 \mathrm{~m} 43 \mathrm{~s}$ & 17313 & 39011 & $9.78 \times 10^{-9}$ & $4.74 \times 10^{-10}$ \\
& 500 & $53 \mathrm{~m} 40 \mathrm{~s}$ & 21106 & 47739 & $9.92 \times 10^{-9}$ & $4.36 \times 10^{-10}$ \\
\hline & 10 & $0.22 \mathrm{~s}$ & 332 & 1557 & $9.84 \times 10^{-9}$ & $1.07 \times 10^{-8}$ \\
& 20 & $0.31 \mathrm{~s}$ & 377 & 1732 & $9.69 \times 10^{-9}$ & $1.11 \times 10^{-8}$ \\
Alg. & 50 & $0.76 \mathrm{~s}$ & 394 & 1402 & $9.51 \times 10^{-9}$ & $9.04 \times 10^{-9}$ \\
3.1 & 100 & $0.81 \mathrm{~s}$ & 387 & 392 & $9.71 \times 10^{-9}$ & $1.76 \times 10^{-9}$ \\
& 150 & $5.13 \mathrm{~s}$ & 886 & 4117 & $9.89 \times 10^{-9}$ & $5.70 \times 10^{-9}$ \\
& 200 & $3.57 \mathrm{~s}$ & 633 & 639 & $9.88 \times 10^{-9}$ & $1.42 \times 10^{-9}$ \\
& 300 & $34.4 \mathrm{~s}$ & 764 & 3137 & $9.82 \times 10^{-9}$ & $8.17 \times 10^{-9}$ \\
& 400 & $02 \mathrm{~m} 19 \mathrm{~s}$ & 1294 & 1300 & $9.90 \times 10^{-9}$ & $9.60 \times 10^{-10}$ \\
& 500 & $03 \mathrm{~m} 11 \mathrm{~s}$ & 1048 & 4442 & $9.98 \times 10^{-9}$ & $4.14 \times 10^{-9}$ \\
\hline
\end{tabular}

TABLE 4

Comparison of Algorithm 3.1 and ode113 for Example 6.2.

\begin{tabular}{|c|r|r|r|r|l|l|}
\hline Alg. & $n$ & CT. & IT. & NF. & Err. & Res. \\
\hline & 10 & $0.37 \mathrm{~s}$ & 1127 & 2394 & $6.88 \times 10^{-9}$ & $1.89 \times 10^{-9}$ \\
& 20 & $1.15 \mathrm{~s}$ & 2487 & 5404 & $7.93 \times 10^{-9}$ & $1.24 \times 10^{-9}$ \\
ode113 & 50 & $6.91 \mathrm{~s}$ & 6019 & 13330 & $9.79 \times 10^{-9}$ & $8.37 \times 10^{-10}$ \\
for & 80 & $20.4 \mathrm{~s}$ & 10338 & 23030 & $9.84 \times 10^{-9}$ & $5.95 \times 10^{-10}$ \\
$(5.4)$ & 100 & $25.9 \mathrm{~s}$ & 9013 & 20186 & $9.76 \times 10^{-9}$ & $6.51 \times 10^{-10}$ \\
& 150 & $01 \mathrm{~m} 09 \mathrm{~s}$ & 10583 & 23655 & $9.70 \times 10^{-9}$ & $6.02 \times 10^{-10}$ \\
& 200 & $02 \mathrm{~m} 13 \mathrm{~s}$ & 11674 & 26119 & $9.64 \times 10^{-9}$ & $5.82 \times 10^{-10}$ \\
& 300 & $07 \mathrm{~m} 47 \mathrm{~s}$ & 13830 & 31022 & $9.98 \times 10^{-9}$ & $5.53 \times 10^{-10}$ \\
& 400 & $22 \mathrm{~m} 49 \mathrm{~s}$ & 17312 & 39008 & $9.93 \times 10^{-9}$ & $4.94 \times 10^{-10}$ \\
& 500 & $46 \mathrm{~m} \mathrm{39} \mathrm{s}$ & 21323 & 48168 & $9.92 \times 10^{-9}$ & $4.51 \times 10^{-10}$ \\
\hline & 10 & $0.19 \mathrm{~s}$ & 330 & 1477 & $9.70 \times 10^{-9}$ & $1.14 \times 10^{-8}$ \\
& 20 & $0.25 \mathrm{~s}$ & 355 & 1341 & $9.72 \times 10^{-9}$ & $7.43 \times 10^{-9}$ \\
Alg. & 50 & $0.86 \mathrm{~s}$ & 440 & 1700 & $9.73 \times 10^{-9}$ & $7.25 \times 10^{-9}$ \\
3.1 & 100 & $1.84 \mathrm{~s}$ & 551 & 1636 & $9.77 \times 10^{-9}$ & $4.22 \times 10^{-9}$ \\
& 150 & $2.04 \mathrm{~s}$ & 505 & 1491 & $9.90 \times 10^{-9}$ & $4.15 \times 10^{-9}$ \\
& 200 & $6.89 \mathrm{~s}$ & 657 & 2530 & $9.94 \times 10^{-9}$ & $6.40 \times 10^{-9}$ \\
& 300 & $5.82 \mathrm{~s}$ & 642 & 647 & $9.99 \times 10^{-9}$ & $1.44 \times 10^{-9}$ \\
& 400 & $03 \mathrm{~m} 12 \mathrm{~s}$ & 1664 & 7780 & $9.74 \times 10^{-9}$ & $8.87 \times 10^{-9}$ \\
& 500 & $03 \mathrm{~m} 14 \mathrm{~s}$ & 1096 & 4805 & $9.97 \times 10^{-9}$ & $6.06 \times 10^{-9}$ \\
\hline
\end{tabular}

For the ODE solver ode113, we evaluate the output values at time intervals of 10. The integration terminates automatically when the above stopping criteria are satisfied. In addition, the other parameters in Algorithm 3.1 are set as above. 
Tables 3 and 4 display the numerical results for Examples 6.1-6.2. We observe from Tables 3 and 4 that the ODE solver ode113 for (3.9) and (5.4) works acceptably for smalland medium-scale problems, while Algorithm 3.1 performs more effectively in terms of computing time.

We must point out the fact that the proposed algorithm converges to different solutions for different initial guesses, and the values of the cost function are almost zero at such different solutions. This can be observed from these and many other numerical tests.

7. Concluding remarks. In this paper, we have considered the doubly stochastic inverse eigenvalue problem (DSIEP) for constructing a doubly stochastic matrix from the given spectral information. We reformulate the inverse problem as a nonlinear least squares problem over several matrix manifolds. By exploiting the Riemannian gradient of the cost function and basic geometric properties (e.g., tangent space, retraction, and vector transport) of these matrix manifolds, we present a Riemannian Fletcher-Reeves conjugate gradient algorithm for the DSIEP. We have established the global convergence of the proposed algorithm. Moreover, we get an extra gain, i.e., our model yields a new Riemannian isospectral flow method. The proposed algorithm is also extended to the DSIEP with prescribed entries. Numerical experiments show that the proposed geometric algorithm is very effective for solving large-scale problems, while our new Riemannian isospectral flow methods work acceptably for smalland medium-scale problems. Since the proposed geometric algorithm converges to different solutions for different initial guesses, an interesting question is how to find an optimal approximation to a given doubly stochastic matrix. This needs further investigation.

Acknowledgments. We would like to thank the editor and the anonymous referees for their valuable comments and suggestions.

\section{REFERENCES}

[1] P.-A. Absil, C. G. Baker, and K. A. Gallivan, A truncated-CG style method for symmetric generalized eigenvalue problems, J. Comput. Appl. Math., 189 (2006), pp. 274-285.

[2] P.-A. Absil, C. G. Baker, And K. A. Gallivan, Trust-region methods on Riemannian manifolds, Found. Comput. Math., 7 (2007), pp. 303-330.

[3] P.-A. Absil And K. A. Gallivan, Joint diagonalization on the oblique manifold for independent component analysis, in Proceedings of the IEEE International Conference on Acoustics, Speech, and Signal Processing (ICASSP), Vol. 5, 2006, pp. V-945-V-948.

[4] P.-A. Absil, R. Mahony, and R. Sepulchre, Optimization Algorithms on Matrix Manifolds, Princeton University Press, Princeton, NJ, 2008.

[5] R. L. Adler, J.-P. Dedieu, J. Y. Margulies, M. Martens, and M. Shub, Newton's method on Riemannian manifolds and a geometric model for the human spine, IMA J. Numer. Anal., 22 (2002), pp. 359-390.

[6] C. G. Baker, P.-A. Absil, And K. A. Gallivan, An implicit Riemannian trust-region method for the symmetric generalized eigenproblem, in Computational Science-ICCS 2006, Lecture Notes in Comput. Sci. 3991, V. N. Alexandrov et al., eds., Springer, New York, 2006, pp. 210-217.

[7] G. BiRkhoff, Three observations on linear algebra, Univ. Nac. Tucumán. Rev. Ser. A, 5 (1946), pp. 147-151.

[8] R. A. Brualdi, Some applications of doubly stochastic matrices, Linear Algebra Appl., 107 (1988), pp. $77-100$.

[9] M. T. ChU AND K. R. Driessel, Constructing symmetric nonnegative matrices with prescribed eigenvalues by differential equations, SIAM J. Math. Anal., 22 (1991), pp. 1372-1387.

[10] M. T. Chu And Q. Guo, A numerical method for the inverse stochastic spectrum problem, SIAM J. Matrix Anal. Appl., 19 (1998), pp. 1027-1039. 
[11] J. E. Cohen, J. H. B. Kemperman, And G. ZbĂGanu, Comparisons of Stochastic Matrices with Applications in Information Theory, Statistics, Economics and Population Sciences, Birkhäuser, Boston, MA, 1998.

[12] M. W. Hinsch, Differential Topology, Graduate Texts in Mathematics 33, Springer, New York, 1976.

[13] S. G. Hwang And S. S. Pyo, The inverse eigenvalue problem for symmetric doubly stochastic matrices, Linear Algebra Appl., 379 (2004), pp. 77-83.

[14] Y. J. LeI, W. R. Xu, Y. Lu, Y. R. Niu, And X. M. GU, On the symmetric doubly stochastic inverse eigenvalue problem, Linear Algebra Appl., 445 (2014), pp. 181-205.

[15] J. D. Louck, Doubly stochastic matrices in quantum mechanics, Found. Phys., 27 (1997), pp. 1085-1104.

[16] M. MARcus, Some properties and applications of doubly stochastic matrices, Amer. Math. Monthly, 67 (1960), pp. 215-221.

[17] L. F. Martignon, Doubly stochastic matrices with prescribed positive spectrum, Linear Algebra Appl., 61 (1984), pp. 11-13.

[18] H. Minc, Nonnegative Matrices, Wiley, New York, 1988.

[19] B. MOURAD, An inverse problem for symmetric doubly stochastic matrices, Inverse Problems, 19 (2003), pp. 821-831.

[20] B. Mourad, On a spectral property of doubly stochastic matrices and its application to their inverse eigenvalue problem, Linear Algebra Appl., 436 (2012), pp. 3400-3412.

[21] B. Mourad, H. Abbas, A. Mourad, A. Ghaddar, and I. Kaddoura, An algorithm for constructing doubly stochastic matrices for the inverse eigenvalue problem, Linear Algebra Appl., 439 (2013), pp. 1382-1400.

[22] A. V. NAZin And B. T. PolyaK, Randomized algorithm to determine the eigenvector of a stochastic matrix with application to the PageRank problem, Autom. Remote Control, 72 (2011), pp. 342-352.

[23] H. Perfect and L. Mirsky, Spectral properties of doubly-stochastic matrices, Monatsh. Math., 69 (1965), pp. 35-37.

[24] S. Petoukhov and M. He, Symmetrical Analysis Techniques for Genetic Systems and Bioinformatics: Advanced Patterns and Applications, IGI Global, Hershey, PA, 2010.

[25] H. SAтo, A Dai-Yuan-type Riemannian conjugate gradient method with the weak Wolfe conditions, Comput. Optim. Appl., to appear.

[26] H. Sato And T. IwaI, A new, globally convergent Riemannian conjugate gradient method, Optimization, 64 (2015), pp. 1011-1031.

[27] O. RoJo AND H. RoJo, Constructing symmetric nonnegative matrices via the fast Fourier transform, Comput. Math. Appl., 45 (2003), pp. 1655-1672.

[28] O. Rojo AND H. Rojo, Some results on symmetric circulant matrices and on symmetric centrosymmetric matrices, Linear Algebra Appl., 392 (2004), pp. 211-233.

[29] E. Seneta, Non-negative Matrices and Markov Chains, Springer, New York, 1981.

[30] M. Shub, Some remarks on dynamical systems and numerical analysis, in Dynamical Systems and Partial Differential Equations, Proceedings of the 7th ELAM, L. Lara-Carrero and J. Lewowicz, eds., pp. 69-92, Equinoccio, Univ. Simón Bolívar, Caracas, 1986.

[31] S. T. Sмiтh, Optimization techniques on Riemannian manifolds, Fields Inst. Commun., 3 (1994), pp. 113-136.

[32] G. W. SoulES, Constructing symmetric nonnegative matrices, Linear Multilinear Algebra, 13 (1983), pp. 241-251.

[33] V. S. Sunder and N. J. Wildberger, Action of finite hypergroups and examples, in Harmonic Analysis and Hypergroups, Bikhoüser, Boston, MA, 1998.

[34] W. R. Xu, Y. J. Lei, X. M. Gu, Y. Lu, And Y. R. Niu, Comment on: "A note on the inverse eigenvalue problem for symmetric doubly stochastic matrices”, Linear Algebra Appl., 439 (2013), pp. 2256-2262.

[35] L. Zhang, W. J. Zhou, And D. H. Li, A descent modified Polak-Ribière-Polyak conjugate gradient method and its global convergence, IMA J. Numer. Anal., 26 (2006), pp. 629-640.

[36] L. Zhang, W. J. Zhou, AND D. H. LI, Global convergence of a modified Fletcher-Reeves conjugate gradient method with Armijo-type line search, Numer. Math., 104 (2006), pp. $561-572$.

[37] Z. ZhaO, Z. J. BAI, AND X. Q. Jin, A Riemannian Newton algorithm for nonlinear eigenvalue problems, SIAM J. Matrix Anal. Appl., 36 (2015), pp. 752-774.

[38] Z. ZhaO, X. Q. Jin, AND Z. J. BAI, A geometric nonlinear conjugate gradient method for stochastic inverse eigenvalue problems, SIAM J. Numer. Anal., submitted.

[39] S. Zhu, T. Gu, AND X. Liu, Solving inverse eigenvalue problems via Householder and rank-one matrices, Linear Algebra Appl., 430 (2009), pp. 318-334.

Copyright $@$ by SIAM. Unauthorized reproduction of this article is prohibited. 